\title{
Synthesis of highly substituted spiropyrrolidines via 1, 3-dipolar cycloaddition reaction of $N$-metalated azomethine ylides. A new access to spiropyrrolines derivatives
}

\author{
Chourouk Mhiri ${ }^{1}$, Fadwa Rouatbi ${ }^{1}$, Sarra Boudriga ${ }^{1}$, Moheddine Askri ${ }^{1, *}$, Kabula Ciamala ${ }^{2}$, Michael Knorr ${ }^{2}$, \\ Karin Monnier-Jobé2, Abderrahim Khatyr ${ }^{2}$, Yoann Rousselin ${ }^{3}$ and Marek M. Kubicki ${ }^{3}$ \\ ${ }^{1}$ Laboratory of Heterocyclic Chemistry Natural Products and Reactivity/LCHPNR, Department of Chemistry, \\ Faculty of Science of Monastir, 5000 Monastir, Tunisia \\ ${ }^{2}$ Institute UTINAM - UMR CNRS 6213, University of Franche-Comté, 16 Route de Gray, \\ F-25030 Besançon, France \\ ${ }^{3}$ Institute of Molecular Chemistry - UMR CNRS 6302, University of Bourgogne, 9 Avenue A. Savary, \\ F-21078 Dijon, France
}

\begin{abstract}
E)$-arylidene-(2H)-indanones $1\left(\mathrm{Ar}=\mathrm{Ph}, p-\mathrm{MeC}_{6} \mathrm{H}_{4}, p\right.$ - $\mathrm{MeOC}_{6} \mathrm{H}_{4}$, $p$ - $\left.\mathrm{ClC}_{6} \mathrm{H}_{4}, p-\mathrm{NO}_{2} \mathrm{C}_{6} \mathrm{H}_{4}\right)$ and $(E)$-2-arylidene- $(2 H)$-tetralones $2\left(\mathrm{Ar}=\mathrm{Ph}, p-\mathrm{MeC}_{6} \mathrm{H}_{4}, p-\mathrm{MeOC}_{6} \mathrm{H}_{4}, p-\mathrm{ClC}_{6} \mathrm{H}_{4}\right.$, $\left.p-\mathrm{NO}_{2} \mathrm{C}_{6} \mathrm{H}_{4}\right)$ to $N$-metalated azomethine ylides 3 generated from methyl $N$-arylideneglycinate in the presence of silver acetate produces in good yields novel methyl 1-oxo-2',4'-diaryl-1,3-dihydrospiro[indene-2,3'-pyrrolidine]5'-carboxylates 4 and methyl 1-oxo-2',4'-diaryl-3,4-dihydro-1H-spiro[naphthalene-2,3'-pyrrolidine]-5'carboxylates $\mathbf{5}$. The cycloaddition proceeds in regio- and stereoselective manner (100\%) at room temperature to afford respectively the syn-endo cycloadducts $\mathbf{4}$ and $\mathbf{5}$ via metallo-azomethine ylides. The regio- and stereochemistry of the spiranic adducts have been established on the basis of spectroscopic data and elemental analysis, corroborated by single-crystal X-ray crystallographic analysis of the heterocycles $\mathbf{4} \mathbf{c i}, \mathbf{4 b g}$ and $\mathbf{5 b i}$. The endo-pyrrolidines 4 were brominated by $N$-bromosuccinimide to give finally the dehydrobrominated 3, 4dihydro- $2 \mathrm{H}$-pyrrole derivatives $\mathbf{6}$. The spiro-adducts $\mathbf{4}$ and their corresponding oxidation products $\mathbf{6}$ are fluorescent in solution.
\end{abstract}

Keywords: 1,3-dipolar cycloaddition; azomethine ylides; spiropyrrolidines; spiropyrrolines; luminescence.

\section{Introduction}

The 1,3-dipolar cycloaddition reaction is one of the best and most useful methods for the construction of five-membered rings in a convergent and stereocontrolled manner. ${ }^{1}$ In particular, the [3+2] cycloaddition of azomethine ylides with alkenes constitutes a direct route to pyrrolidine derivatives ${ }^{1-3}$, which are valuable substrates in synthetic organic chemistry ${ }^{4,5}$, pharmacology ${ }^{6}$ and biology. ${ }^{7-9}$

Among the different versions of this reaction, one of most practical approach is the interaction between stabilized $N$-metalated azomethine ylides and electron-deficient alkenes $^{5,9}$.

*Corresponding author: Moheddine Askri

E-mail address: moheddine.askri@fsm.rnu.tn

DOI: http://dx.doi.org/10.13171/mjc.4.1.2015.18.02.09.52/askri 
This method allows the cycloaddition to proceed under mild reaction conditions with a high degree of diastereocontrol ${ }^{10}$. Silver salts are the most commonly used catalyst to facilitate the reaction along with an excess of base such as a tertiary amine ${ }^{11}$.

Spiropyrrolidines have attracted much attention because of their antiproliferative $\mathrm{e}^{12}$ and antituberculosis activities ${ }^{13}$, as well as potential antileukemic and anticonvulsant agents ${ }^{14}$.

Moreover, many indanone and tetralone derivatives have been used as versatile intermediates for the synthesis of several natural and pharmaceutical products ${ }^{15-17}$. The combination of this chemical entity with a pyrrolidine unit might as well be envisioned to conceive heterocyclic compound with potentially interesting biological properties.

Recently, several reports have appeared on the synthesis of substituted spiropyrrolidines using azomethine ylides cycloaddition reactions ${ }^{18}$. For example, the work of Wang and co-workers ${ }^{19}$ employing a silver acetate/(S)-TF-Biphamphos complex as catalyst afforded an elegant access to spiroheterocyclic compounds containing the pyrrolidine motif. Since several years, we have focused our studies on the reactivity of dipolarophiles bearing an exocyclic carbon-carbon double bond towards several 1,3-dipoles such as nitrones ${ }^{20}$, nitrile oxides ${ }^{21}$, diazoalkanes $^{22}$ and azomethine ylides ${ }^{23}$. We particularly paid attention to the role played by the substituents of the dipolarophile and dipole entities on chemical reactivity, regio- and stereoselectivity.

In continuation of our research interest in this field, we present in this contribution the synthesis of spiropyrrolidine derivatives through regio- and diastereoselective cycloaddition of $(E)$-2-arylidene- $(2 H)$-indanones 1a-e and $(E)$-2-arylidene- $(2 H)$-tetralones 2a-e with $N$ metalated azomethine ylides generated in situ by deprotonation of the corresponding iminoesters 3f-i derived from glycine methyl ester. The reaction affords spiropyrrolidine derivatives 4af-ei and 5af-ei, respectively, with high regio- and stereoselectivity. In order to assess the impact of the substituent at the $p$-position of dipolarophiles and azomethine ylides on the outcome of the reaction, we examined the influence of the electronic and steric effects exerted by the aryl ring substituents. Furthermore, the products 4 were transformed by action of NBS to methyl 1-oxo-2',4'-diaryl-1,3,4',5'-tetrahydrospiro[indene-2,3'-pyrrole]-5'carboxylate derivatives 6 . Finally, we report on the luminescence properties of some selected compounds.

\section{Results and Discussion}

\section{Synthesis of the spiroheterocycles}

The (E)-2-arylidene- $(2 H)$-indanones 1a-e and (E)-2-arylidene- $(2 H)$-tetralones 2a-e employed as dipolarophiles have been prepared by the acid-catalyzed condensation of indanone and tetralone with various benzaldehydes. As confirmed by NMR spectroscopy, these starting materials display an E-configuration in accordance with our earlier studies and the literature ${ }^{24}$.

The methyl $\mathrm{N}$-arylideneglycinate were prepared by condensation of appropriately substituted aromatic aldehydes Ar'CHO with methyl glycine ester according to reported methods ${ }^{25-27}$.

Initially, we studied the cycloaddition reaction without catalyst, choosing methyl $N$-chlorobenzylidene glycinate $\left(\mathrm{Ar}^{\prime}=p-\mathrm{ClC}_{6} \mathrm{H}_{4}\right) 3 \mathbf{i}$ and exocyclic enones $\mathbf{1 a}$ and $\mathbf{2 a}(\mathrm{Ar}=\mathrm{Ph})$ as reagents. The outcome was however very unsatisfying. Whatever the solvent used, both at room temperature or under reflux during seven days, the obtained yields were always less than $10 \%$. In order to optimize these conditions, we found that $\mathrm{Ag}_{2} \mathrm{CO}_{3}, \mathrm{Ag}_{2} \mathrm{O}$ and $\mathrm{AgOAc}$ 
catalysed the 1,3-dipolar cycloaddition reaction affording the desired spiropyrrolidine in $60 \%, 64 \%$, and 70\% yield, respectively (Table 1, entries 1-3) in toluene as solvent. Then, using $\mathrm{AgOAc}$ as catalyst, various solvents were screened, and it was found that $\mathrm{CH}_{2} \mathrm{Cl}_{2}$ and $\mathrm{CH}_{3} \mathrm{CN}$ were the most appropriate solvents for the reaction with $\mathbf{1}$ and $\mathbf{2}$, respectively (Table 1, entries 4-7).

Table 1. Optimization of the reaction condition ${ }^{\mathrm{a}}$

\begin{tabular}{|c|c|c|c|c|c|c|}
\hline Entry & Dipolarophile & Product & $\begin{array}{c}\text { Catalyst } \\
(10 \mathrm{~mol} \%) \\
\end{array}$ & Solvent & Time (h) & Yields $(\%)^{\mathrm{b}}$ \\
\hline 1 & $1 \mathrm{a}$ & 4ai & $\mathrm{Ag}_{2} \mathrm{CO}_{3}$ & Toluene & 48 & 60 \\
\hline 2 & $1 \mathrm{a}$ & 4ai & $\mathrm{Ag}_{2} \mathrm{O}$ & Toluene & 36 & 64 \\
\hline 3 & $1 \mathrm{a}$ & 4ai & AgOAc & Toluene & 12 & 70 \\
\hline 4 & $1 \mathrm{a}$ & 4ai & $\mathrm{AgOAc}$ & $\mathrm{CH}_{3} \mathrm{CN}$ & 6 & 72 \\
\hline 5 & $1 \mathrm{a}$ & 4ai & $\mathrm{AgOAc}$ & $\mathrm{CH}_{2} \mathrm{Cl}_{2}$ & 2 & 80 \\
\hline 6 & $2 \mathrm{a}$ & $5 \mathrm{ai}$ & $\mathrm{AgOAc}$ & $\mathrm{CH}_{3} \mathrm{CN}$ & 4 & 77 \\
\hline 7 & $2 \mathrm{a}$ & $5 \mathrm{ai}$ & $\mathrm{AgOAc}$ & $\mathrm{CH}_{2} \mathrm{Cl}_{2}$ & 6 & 75 \\
\hline
\end{tabular}

Having established suitable reaction conditions, we next explored the scope and generality of this methodology (Scheme 1). The AgOAc catalyzed [3+2] cycloadditions of (E)-2arylidene- $(2 H)$-indanones $\mathbf{1}$ and $(E)$-2-arylidene- $(2 H)$-tetralones $\mathbf{2}$ with iminoesters $\mathbf{3}$ led to unique adducts corresponding to the expected novel spiropyrrolidine derivatives, the syn-endo methyl 1-oxo-2',4'-diaryl-1,3-dihydrospiro[indene-2,3'-pyrrolidine]-5'-carboxylates $\mathbf{4}$ and methyl 1-oxo-2',4'-diaryl-3,4-dihydro-1H-spiro[naphthalene-2,3'-pyrrolidine]-5'-carboxylates 5. The reactions proceed at room temperature with $100 \%$ regioselectivity.

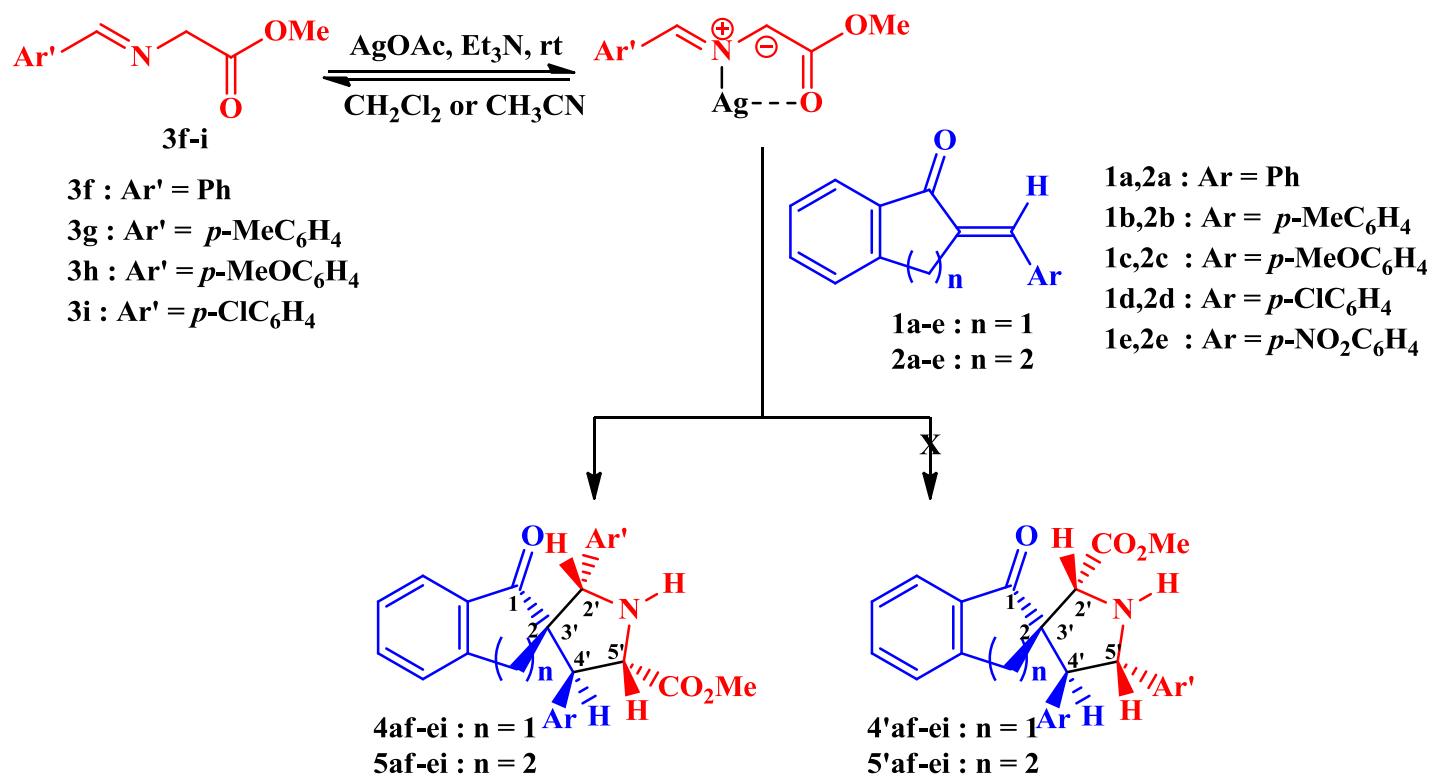

Scheme 1. Reactions of enones $\mathbf{1}$ and $\mathbf{2}$ with methyl $N$-arylideneglycinate $\mathbf{3}$

All results reported below proof that pyrrolidines were obtained with conservation of the stereochemistry of the starting alkenes. The structure and stereochemistry of the spiropyrrolidine resulting from the cycloaddition has been established on the basis of 
elemental analyses, spectroscopic data and three X-ray structure determinations performed on cycloadducts $\mathbf{4 c i}, \mathbf{4 b g}$ and $\mathbf{5 b i}$.

\section{Spectroscopic characterisation}

The IR spectra of the compounds $\mathbf{4}$ and $\mathbf{5}$ contain absorption bands at around $3447 \mathrm{~cm}^{-1}$, $1723 \mathrm{~cm}^{-1}$, and $1670 \mathrm{~cm}^{-1}$ due to $\mathrm{NH}, \mathrm{C}=\mathrm{O}$ ester and $\mathrm{C}=\mathrm{O}$ ring stretching vibrations, respectively. In their ${ }^{1} \mathrm{H}$ NMR spectra, we observed a singlet at 4.23-4.62 ppm and a doublet in the range between 4.40-4.68 ppm due to benzylic hydrogens H-2' and H-4', respectively, which clearly ascertain the regiochemistry of the cycloaddition reaction in accordance with the literature. $^{9 \text { b, } 28,29}$ The two protons $\mathrm{H}-5$ ' and $\mathrm{H}-4$ ' couple with each other with $J$ values ranging between 5.7 and $6.3 \mathrm{~Hz}$ in the case of compounds 4 , and between 6.9 and $8.7 \mathrm{~Hz}$, for cycloadducts $\mathbf{5}$, suggesting them to be on trans configuration. Another characteristic in products $\mathbf{4}$ and $\mathbf{5}$ is the presence of an $\mathrm{N}-\mathrm{H}$ resonance between 2.49 and $3.55 \mathrm{ppm}$, exchangeable by $\mathrm{D}_{2} \mathrm{O}$.

The ${ }^{1} \mathrm{H}$ and ${ }^{13} \mathrm{C}$ NMR spectra of the spiro-adducts $\mathbf{4}$ and $\mathbf{5}$ exhibited only one set of signals, thereby confirming the formation of single diastereoisomer during the cycloaddition reactions. The stereochemistry of the cycloadducts $\mathbf{4}$ and $\mathbf{5}$ is based on the usual facial selectivity and endo-transition state observed. No cycloadduct corresponding to an exo-transition state has been evidenced. Thus in all the cases, the products $\mathbf{4}$ and $\mathbf{5}$ are formed via an endo-transition state involving $E, E$-(syn-dipole). This could be explained by the endo-transition state shown in Figure 1, where both the ylide and the dipolarophile coordinate to the $\mathrm{Ag}$ ion via two carbonyl $\mathrm{Ag}$... O interactions and an $\mathrm{Ag}-\mathrm{N}$ bond, whereas just one $\mathrm{Ag}$... O and $\mathrm{Ag}-\mathrm{N}$ interaction is present in the exo-transition state.

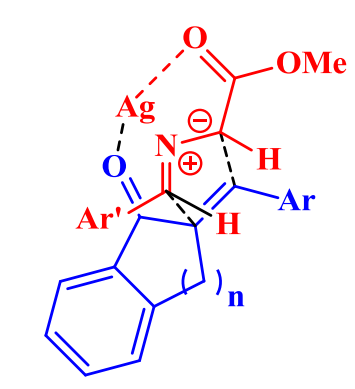

endo transition state

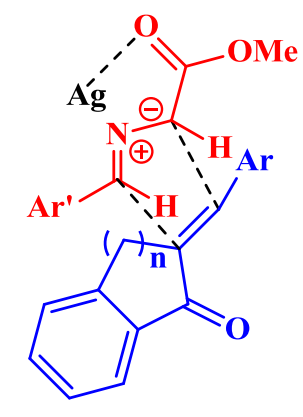

exo transition state

Figure 1. Proposed transition states for the cycloaddition of enones $\mathbf{1}$ and $\mathbf{2}$ with azomethine ylide

\section{Crystallographic characterisation.}

The relative stereochemistry of the cycloadducts $\mathbf{4}$ and $\mathbf{5}$ was furthermore confirmed by X-ray crystal analysis of 4ci, $\mathbf{4 b g}$ and 5bi (Figures 2, 3 and 4), showing that the ester carbonyl and aryl group stemming from the dipolarophile are in trans configuration. The crystal data collection and structure refinement of $\mathbf{4 c i}, \mathbf{4 b g}$ and $5 \mathbf{b i}$ are summarized in Table 2. 


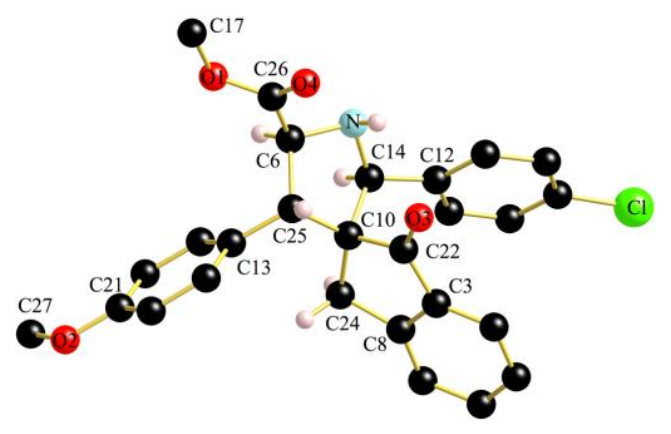

Figure 2. Molecular structure of spirocompound 4ci. Selected bond lengths $(\AA)$ and angles $\left({ }^{\circ}\right)$. The aromatic hydrogen atoms have been omitted for clarity: C26-O4 1.202(3), N-C6 1.469(3), C6-C25 1.575(4), N-C14 1.462(3), C14-C10 1.596(4), C10-C25 1.563(3), C10-C22 1.529(3), O3-C22 1.222(3), C22C3 1.466(4), C3-C8 1.395(4); C26-C6-N 109.6(2), C6-N-C14 101.9(2), N-C14-C10 115.1(2), N-C14-C12 114.9(2), N-C6-C25 106.4(2), C25-C10-C14 102.55(19), C25-C10-C24 119.6(2), C25-C10-C22 111.6(2), C24-C10-C22 104.3(2), C10-C22-O3 124.3(2), O3-C10-C3 127.6(2), C22-C3-C8 109.3(2), C3-C8-C24 $111.0(2)$.

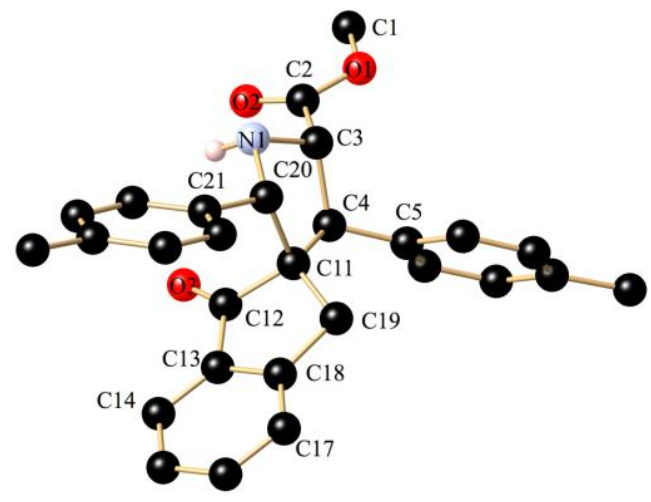

Figure 3. Molecular structure of spirocompound $\mathbf{4 b g}$. Selected bond lengths $(\AA)$ and angles $\left(^{\circ}\right)$ : C1-O2 1.203(2), N-C3 1.465(2), C3-C4 1.570(2), N-C20 1.461(2), C20-C11 1.593(2), C11-C4 1.566(2), $\mathrm{C} 11-\mathrm{C} 12$ 1.526(2), O2-C12 1.223(2); C2-C3-N 109.83(14), C3-N1-C20 102.33(13), N1-C20-C21 114.08(14), N1-C20-C11 105.09(13), C20-C11-C4 102.79(13), C20-C11-C12 106.28(13), C20-C11-C19 110.99(13), C11-C12-O3 124.89(15), O3-C12-C13 127.24(16), C12-C13-C18 108.93(15), C12-C11-C19 104.28(13).

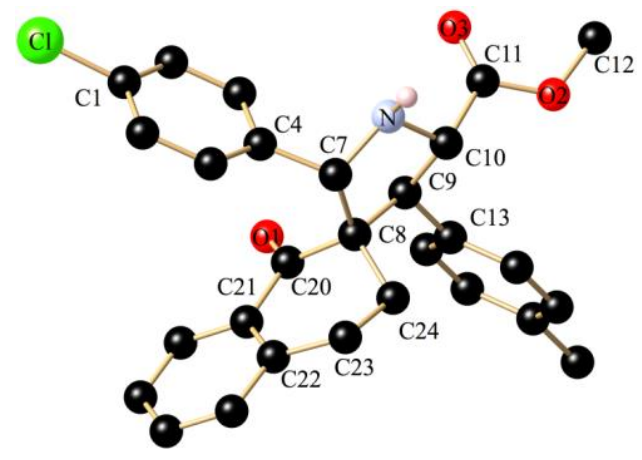

Figure 4. Molecular structure of spirocompound 5bi. Selected bond lengths $(\AA)$ and angles $\left({ }^{\circ}\right)$ : C11-O3 1.198(2), C11-C10 1.514(4), N-C10 1.455(3), N-C7 1.479(3), C7-C8 1.585(3), C8-C9 1.560(3), C11-C4 1.566(2), C9-C10 1.537(3), C8-C20 1.523(4), O1-C20 1.225(3), C21-C20 1.494(4); C4-C7-N 109.6(2), C7-N-C10 109.9(2), N-C10-C9 114.08(14), N-C7-C8 104.32(19), C7-C8-C9 102.3(2), C8-C9-C10 101.6(2), C7-C8-C20 111.6(2), C20-C8-C24 109.1(2), C8-C20-O1 121.5(2), O1-C20-C21 121.1(2), C24C8-C9 112.5(2).

The three compounds studied by X-ray diffraction apparently don't need any further strong intermolecular interaction for stabilization of their crystal phases. One could expect the presence of intermolecular $\mathrm{N}-\mathrm{H} \ldots \mathrm{O}(=\mathrm{C})$ hydrogen bonds. However, there is almost no 
occurrence of this bonding interaction in the three compounds. It's only in $\mathbf{5 b i}$, where the electronegative atoms $\mathrm{N}, \mathrm{O}$ and $\mathrm{Cl}$ are involved in a very weak and bifurcated "hydrogen bond" interaction (Figure S1 in ESI). The N-H...O (ketone), C-H...O (ketone) and C$\mathrm{H}$...Cl interactions seem to be of similar strength therein. The crystal packing in $\mathbf{4 b g}$ is assured only by a very weak C-H...C (different nature) interactions (Figure S2 in ESI) and by the $\mathrm{C}-\mathrm{H}$... O (ketone and carboxylate) ones in 4ci (Figure S3 in ESI). In conclusion, the intermolecular contacts within these three crystal structures are mainly built from weak van der Waals interactions that are of different nature in each individual case.

Table 2. Crystal data collection and structure refinement of $\mathbf{4 c i}, \mathbf{4 b g}$ and $\mathbf{5 b i}$.

\begin{tabular}{|c|c|c|c|}
\hline Compound /Formula & 4bg $/ \mathrm{C}_{28} \mathrm{H}_{27} \mathrm{NO}_{3}$ & 4ci / $\mathrm{C}_{27} \mathrm{H}_{24} \mathrm{ClNO}_{4}$ & 5bi / $\mathrm{C}_{28} \mathrm{H}_{26} \mathrm{ClNO}_{3}$ \\
\hline Formula weight & 425.51 & 461.92 & 459.95 \\
\hline Temperature/K & $115(2)$ & $115(2)$ & $115(2)$ \\
\hline Wavelength/ & 0.71073 & 0.71073 & 0.71069 \\
\hline Crystal system & Monoclinic & Monoclinic & Monoclinic \\
\hline Space group & $\mathrm{P} 2{ }_{1} / \mathrm{c}$ & $\mathrm{P} 2{ }_{1} / \mathrm{c}$ & $\mathrm{P} 2{ }_{1} / \mathrm{c}$ \\
\hline$a / \AA$ & $12.2869(3)$ & $15.7976(4)$ & $13.3588(5)$ \\
\hline$b / \AA$ & $11.7559(3)$ & $6.4703(2)$ & $12.4090(5)$ \\
\hline$c / \AA$ & $16.7554(4)$ & $22.7657(8)$ & $16.6956(6)$ \\
\hline$\beta$ & $108.7240(10)$ & $103.638(2)^{\circ}$ & $125.314(2$ \\
\hline Volume/ $\AA^{3}$ & $2292.12(10)$ & $2261.39(12)$ & $3627.3(2)$ \\
\hline$Z$ & 4 & 4 & 4 \\
\hline Density (calculated) $\mathrm{g} / \mathrm{cm}^{3}$ & 1.233 & 1.357 & 1.353 \\
\hline Absorp. coefficient $/ \mathrm{mm}^{-1}$ & 0.080 & 0.204 & 0.201 \\
\hline$F(000)$ & 904 & 968 & 968 \\
\hline Crystal size $/ \mathrm{mm}^{3}$ & $0.40 \times 0.40 \times 0.40$ & $0.12 \times 0.07 \times 0.02$ & $0.20 \times 0.10 \times 0.10$ \\
\hline $\begin{array}{c}\text { Theta range for data } \\
\text { collection }{ }^{\circ}\end{array}$ & 3.03 to 27.50 & 3.28 to 27.51 & 2.99 to 27.47 \\
\hline Index ranges & $\begin{array}{c}-15<=\mathrm{h}<=15, \\
-15<=\mathrm{k}<=9, \\
-21<=\mathrm{l}<=21\end{array}$ & $\begin{array}{c}-20<=\mathrm{h}<=20, \\
-8<=\mathrm{k}<=8, \\
-29<=1<=29\end{array}$ & $\begin{array}{c}-17<=\mathrm{h}<=17, \\
-16<=\mathrm{k}<=11, \\
-21<=\mathrm{l}<=21\end{array}$ \\
\hline Reflections collected & 7848 & 9394 & 7276 \\
\hline Independent reflections & $\begin{array}{c}5215[R(\mathrm{int})= \\
0.0177]\end{array}$ & $\begin{array}{c}5127[\mathrm{R}(\mathrm{int})= \\
0.0568]\end{array}$ & $\begin{array}{c}5084[\mathrm{R}(\mathrm{int})= \\
0.0280]\end{array}$ \\
\hline Refinement method & $\begin{array}{c}\text { Full-matrix least- } \\
\text { squares on } F^{2} \\
\end{array}$ & $\begin{array}{c}\text { Full-matrix least- } \\
\text { squares on } F^{2}\end{array}$ & $\begin{array}{c}\text { Full-matrix least- } \\
\text { squares on } F^{2}\end{array}$ \\
\hline Data/restraints/parameters & $5215 / 1 / 295$ & $5127 / 0 / 303$ & $5084 / 0 / 300$ \\
\hline Goodness-of-fit on $F^{2}$ & 1.029 & 1.111 & 1.108 \\
\hline $\begin{array}{c}\text { Final } R \text { indices } \\
{[I>2 \operatorname{sigma}(\mathrm{I})]}\end{array}$ & $\begin{array}{c}R 1=0.0572, \\
\mathrm{w} R 2=0.1218\end{array}$ & $\begin{array}{c}R 1=0.0659, \\
\mathrm{w} R 2=0.1133\end{array}$ & $\begin{array}{c}R 1=0.0636, \\
\mathrm{w} R 2=0.1161\end{array}$ \\
\hline$R$ indices (all data) & $\begin{array}{c}R 1=0.0714, \\
\mathrm{w} R 2=0.1328\end{array}$ & $\begin{array}{c}R 1=0.0972, \\
\mathrm{w} R 2=0.1274\end{array}$ & $\begin{array}{c}R 1=0.0917, \\
\mathrm{w} R 2=0.1333\end{array}$ \\
\hline $\begin{array}{c}\text { Largest diff. peak and } \\
\text { hole/e. } \AA^{-3}\end{array}$ & 0.339 and -0.485 & 0.321 and -0.305 & 0.339 and -0.337 \\
\hline
\end{tabular}




\section{Treatment of 4 with $N$-bromosuccinimide (NBS)}

The methyl 1-oxo-2',4'-diaryl-1,3-dihydrospiro[indene-2,3'-pyrrolidine]-5'-carboxylates 4ag, 4ah and 4df were allowed to react at room temperature with $N B S$ in $\mathrm{CH}_{2} \mathrm{Cl}_{2}$ for $1 \mathrm{~h} 30^{30}$. The formation of the single methyl 1-oxo-2',4'-diaryl-1,3,4',5'-tetrahydrospiro[indene-2,3'pyrrole]-5'-carboxylate in each case suggests the reaction first involves the bromination of the amine followed by the spontaneous elimination of hydrogen bromide to afford the corresponding methyl 1-oxo-2',4'-diaryl-1,3,4',5'-tetrahydrospiro[indene-2,3'-pyrrole]-5'carboxylates 6. The elimination of the hydrogen bromide was facilitated by the highly conjugated nature of the newly formed double bond (Scheme 2). The structure of these compounds was established from elemental analyses and spectroscopic data. In the ${ }^{1} \mathrm{H}$ NMR spectra of compounds 6 appear two doublets between 4.10-4.30 ppm and 4.9-5.15 ppm corresponding to the $\mathrm{H}-4$ ' and $\mathrm{H}-5$ ' protons, respectively. This excludes the formation of other isomer ' 6 ' bearing the imine function between the $\mathrm{N}$-atom and $\mathrm{C}-5$ '. In this case, the protons H-2' and H-4' should appear as singlets. The ${ }^{13} \mathrm{C}$ NMR spectrum shows a signal at $139 \mathrm{ppm}$ corresponding to the carbon atom of imine group. This was corroborated by the presence of a $\mathrm{C}=\mathrm{N}$ vibration at $1640 \mathrm{~cm}^{-1}$ in the infrared spectra. These data are in favour of elimination of H-5 proton during the dehydrobromination of spiropyrrolidines 4 .
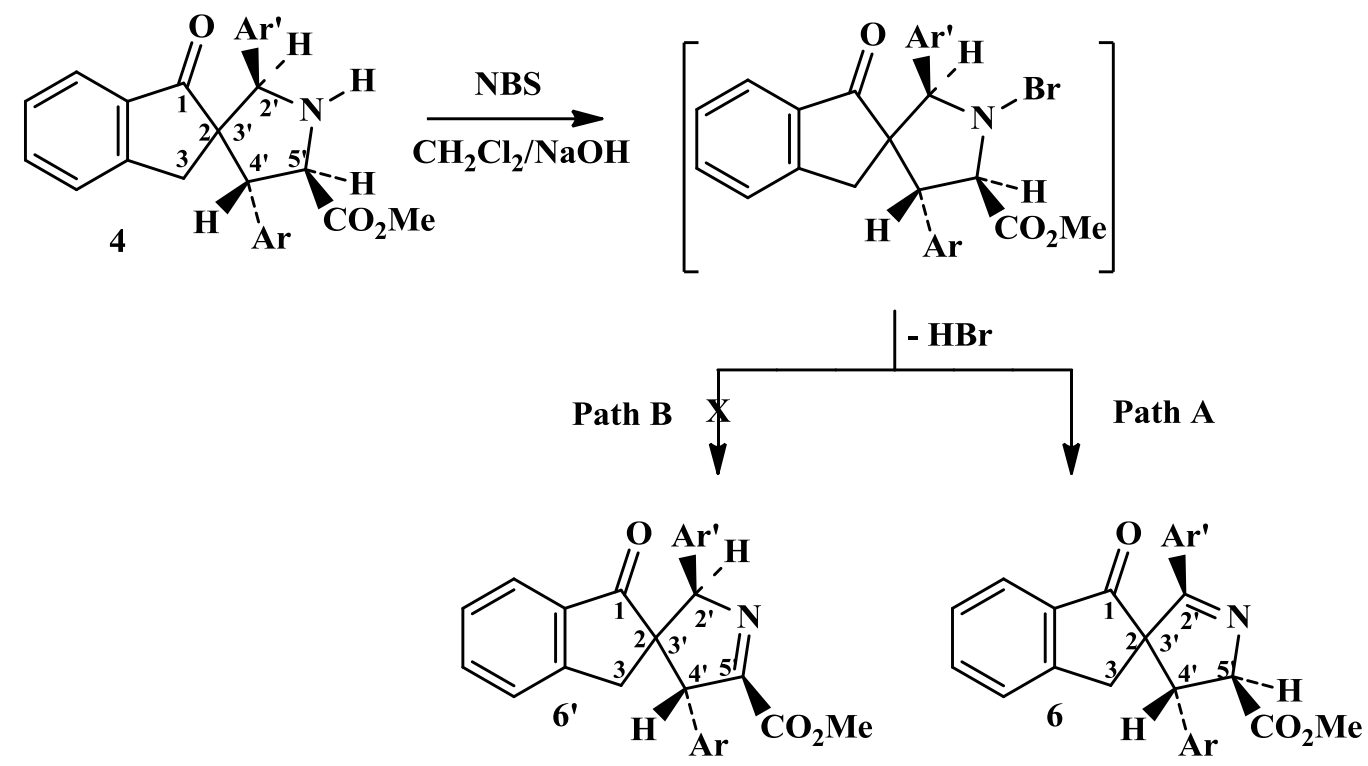

Scheme 2. Dehydrobromination of spiropyrrolidines 4 with $N B S$

\section{UV-vis spectra and luminescence properties}

The electronic absorption and emission spectra of compounds 4, 5 and $\mathbf{6}$ have been recorded at $298 \mathrm{~K}$ using $\mathrm{CH}_{2} \mathrm{Cl}_{2}$ as solvent. Figures $\mathbf{5}$ and $\mathbf{6}$ show representative spectra of compounds $4 \mathbf{b g}$ and $\mathbf{6 a g}$, respectively. The absorption and emission data obtained from all compounds are summarized in Table 3. The electronic absorption spectra of these compounds in general exhibit a strong band with a maximum between 230 and $280 \mathrm{~nm}$ attributed to $\pi, \pi^{*}$ transitions and a low-intensity band in the spectral range from 280 to $380 \mathrm{~nm}$ assigned to a $\mathrm{n}, \pi^{*}$ transition. After excitation at $270 \mathrm{~nm}$, compounds 4 exhibit emission maxima around $370 \mathrm{~nm}$. However, no emission is observed for compounds 5. Compounds $\mathbf{6}$ show the emission maxima in the similar spectral rang as observed for compounds $\mathbf{4}$ and other spiropyrrolidines published in the literature. ${ }^{31}$ This study revealed that this emission bands are independent of the excitation wavelength. These fluorescence bands can be assigned to the 
lowest energy singlet state $\mathrm{S}_{1} \rightarrow \mathrm{S}_{0}$ transition. The only exception is observed for compound 4ef which features two emission bands with maxima centred at 371 and $435 \mathrm{~nm}$. We attribute this lowest energy to a charge transfer band due to the high sensitivity of emission wavelength to the solvent polarity $\lambda_{\max }=398\left(\mathrm{C}_{6} \mathrm{H}_{12}\right), 406(\mathrm{EtOH})$ and $557 \mathrm{~nm}(\mathrm{MeCN})$. Fluorescence quantum yields $\varnothing_{\mathrm{F}}$ of compounds $\mathbf{4}$ and $\mathbf{6}$ were determined using cresyl violet as a fluorescence quantum yield standard ${ }^{32}$. All in all, these values are lower compared to spirobased anthracenone compounds ${ }^{33 a}$, but they exhibit quantum yields relatively similar to those of the family of isatin-based spiro compounds reported in the literature ${ }^{33 b}$.

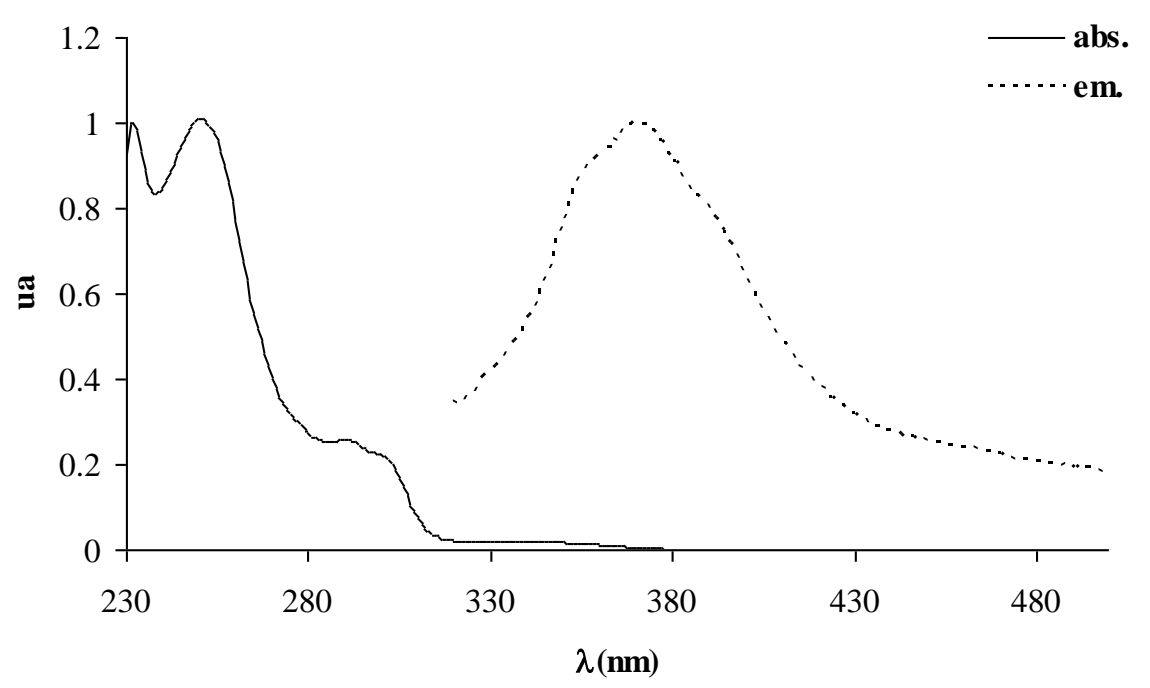

Figure 5. Normalized absorption (solid curve), and emission (dashed curve) spectra recorded for $4 \mathrm{bg}$ in $\mathrm{CH}_{2} \mathrm{Cl}_{2}$ at $293 \mathrm{~K}$.

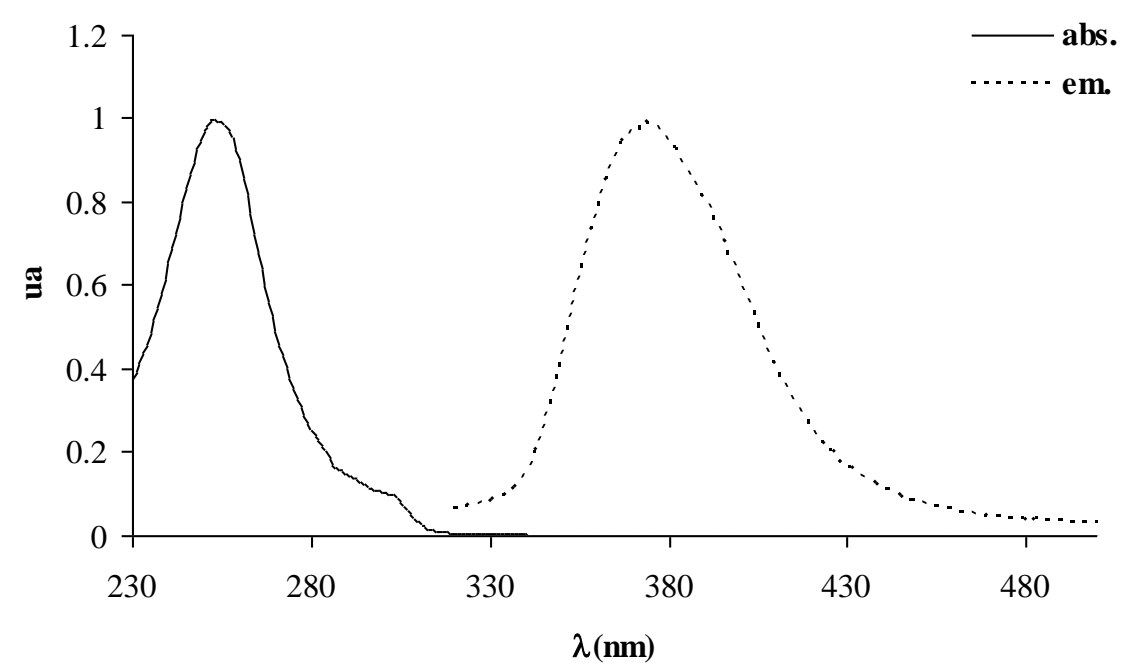

Figure 6. Normalized absorption (solid curve), and emission (dashed curve) spectra recorded for $6 \mathrm{ag}$ in $\mathrm{CH}_{2} \mathrm{Cl}_{2}$ at $293 \mathrm{~K}$. 
Table 3. Absorption and emission data of compounds 4, 5 and 6. a) Values of absorption maxima $\left(\lambda_{\text {abs. }}\right)$ and their associated molar extinction coefficient $(\varepsilon)$; b) $\lambda_{\text {excit. }}$ : Excitation wavelength; c) $\lambda_{\mathrm{em}}$. Emission wavelength; d) $\phi$ : Fluorescence quantum yield $( \pm 8 \%)$.

\begin{tabular}{|c|c|c|c|}
\hline \multirow[b]{2}{*}{ Compound } & \multirow{2}{*}{$\begin{array}{c}\text { Absorption } \\
\mathrm{CH}_{2} \mathrm{Cl}_{2} \text { at } 298 \mathrm{~K} \\
\lambda_{\text {abs }}(\mathrm{nm})\left[\varepsilon\left(\mathrm{M}^{-1} \mathrm{~cm}^{-1}\right)\right]\end{array}$} & \multicolumn{2}{|l|}{ Emission } \\
\hline & & $\begin{array}{c}\mathrm{CH}_{2} \mathrm{Cl}_{2} \text { at } 298 \mathrm{~K} \\
\lambda_{\text {em }}(\mathrm{nm})\left[\lambda_{\text {excit }}=270 \mathrm{~nm}\right]\end{array}$ & $\emptyset_{\mathrm{F}}$ \\
\hline 4ai & $\begin{array}{c}230 \text { [9890], } 254 \text { [8040], } \\
294 \text { sh [1700] }\end{array}$ & 370 & 0.4 \\
\hline $4 b g$ & $\begin{array}{c}232[9770], 253[9680], \\
294 \operatorname{sh}[2400], 346[190]\end{array}$ & 370 & 0.5 \\
\hline $4 \mathrm{ef}$ & $\begin{array}{c}257[10180], 284[10400], \\
345 \mathrm{sh}[1700]\end{array}$ & 371,435 & 0.7 \\
\hline $5 \mathrm{ag}$ & $\begin{array}{c}232[9790], 253[9700], \\
294 \operatorname{sh}[2420], 346[210]\end{array}$ & $*$ & $*$ \\
\hline 5 bi & $\begin{array}{c}234[10700], 256[13000], \\
296 \mathrm{sh}[1950]\end{array}$ & $*$ & $*$ \\
\hline $5 \mathrm{dg}$ & $\begin{array}{c}231[10250], 257 \text { [6950], } \\
296 \mathrm{sh}[1320]\end{array}$ & * & $*$ \\
\hline 6ag & 253 [14150], $304 \mathrm{sh}[1260]$ & 373 & 0.8 \\
\hline $6 \mathrm{ah}$ & $\begin{array}{l}235 \operatorname{sh}[10000], 253 \text { [13000], } \\
292 \operatorname{sh}[1950], 304 \operatorname{sh}[1200]\end{array}$ & 369 & 0.6 \\
\hline
\end{tabular}

*No emission

\section{Conclusion}

In conclusion, we have shown that the cycloaddition reaction of enones (1a-e) and (2a-e) with azomethine ylides $\mathbf{3 f - i}$ leads straightforwardly to novel spiropyrrolidines in a regio- and diastereoselective manner (100\%). The regiochemistry of the reaction is not influenced by the electronic nature of the substituents at the para-position on the dipolarophile as well as on the dipole. The cycloadducts $\mathbf{4}$ and $\mathbf{5}$ are formed exclusively via an endo-transition state involving $E$,E-(syn-dipole). Spiropyrrolidines 4 reacts under mild condition with $N B S$ in $\mathrm{CH}_{2} \mathrm{Cl}_{2}$ to afford the corresponding methyl 1-oxo-2',4'-diaryl-1,3,4',5'-tetrahydrospiro[indene-2,3'pyrrole]-5'-carboxylates 6. Finally, the study of the luminescence properties of some compounds revealed that spiro-adducts $\mathbf{4}$ and their corresponding oxidation products 6 are fluorescent in solution at room temperature.

\section{Experimental Section}

Reactions were carried out under an atmosphere of dry $\mathrm{N}_{2}$. Solvents were purified by standard methods and freshly distilled under nitrogen and dried before use. Melting points were determined on a Kofler bank. Materials: thin-layer chromatography (TLC): TLC plates

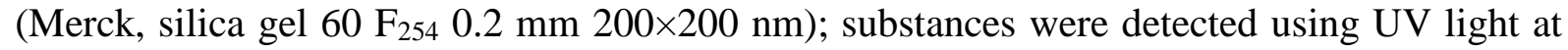
$254 \mathrm{~nm} .{ }^{1} \mathrm{H},{ }^{13} \mathrm{C}$ NMR spectra were recorded on a Bruker $300 \mathrm{MHz}$ instrument in $\mathrm{CDCl}_{3}$ using TMS as an internal standard. Chemical shifts are given in parts per million (d-scale) and the coupling constants are given in Hertz. IR spectra were recorded on a JASCOFT IR instrument ( $\mathrm{KBr}$ pellet in case of solids). UV-vis spectra were measured in $\mathrm{CH}_{2} \mathrm{Cl}_{2}\left(\mathrm{c} \sim 10^{-5} \mathrm{M}\right)$ at room temperature with a VARIAN-Cary 100 spectrophotometer. Emission spectra were 
recorded in $\mathrm{CH}_{2} \mathrm{Cl}_{2}\left(\mathrm{c} \sim 10^{-6} \mathrm{M}\right)$ at room temperature on a Jobin-Yvon FluoroLog 3.2.2 apparatus using a $1 \mathrm{~cm}$ width quartz cell with a scan speed of $1 \mathrm{~nm} / \mathrm{s}$ and a slit width of 5 . The enones (1a-e) and (2a-e) are obtained by condensation of aldehydes ArCHO with indanone and tetralone, respectively, in the presence of $p$-toluenesulfonic acid (APTS) according to ref ${ }^{24}$, the methyl $\mathrm{N}$-arylideneglycinate were prepared from reaction of aromatic aldehydes Ar'CHO with methyl glycine ester according to reported methods ${ }^{25-27}$.

\section{General procedure for the cycloaddition reaction of enones 1 and 2 with azomethine ylide}

To a solution of methyl $\mathrm{N}$-arylideneglycinate (3f-i) $(1 \mathrm{mmol})$ in dry $\mathrm{CH}_{2} \mathrm{Cl}_{2}$ or $\mathrm{CH}_{3} \mathrm{CN}$ (10 $\mathrm{mL})$, triethylamine $(1 \mathrm{mmol})$, enone (1a-e) or (2a-e) (1 mmol) and $\mathrm{AgOAc}(0.10$ equiv) were added. After completion of the reaction as determined by TLC, the reaction mixture was filtered through a celite pad, washed with saturated aqueous solution of $\mathrm{NH}_{4} \mathrm{Cl}$ and then extracted with $\mathrm{CH}_{2} \mathrm{Cl}_{2}$ The combined organic layers was washed with brine, dried $\left(\mathrm{MgSO}_{4}\right)$ filtered and the solvent evaporated in vacuo. The crude product was purified by column chromatography on silica gel using cyclohexane/ethyl acetate $(7: 3)$ as the eluent to give analytical pure spiropyrrolidines $\mathbf{4}$ and $\mathbf{5}$.

\section{Preparation of spiropyrrolines 6}

To a stirred solution of cycloadducts $\mathbf{4 a g}$, $\mathbf{4 a h}$ and $\mathbf{4 d f}(0.75 \mathrm{mmol})$ in $\mathrm{CH}_{2} \mathrm{Cl}_{2}(2 \mathrm{~mL})$ was added portion wise of $N B S(0.14 \mathrm{~g}, 0.82 \mathrm{mmol})$ at room temperature over $20 \mathrm{~min}$ and stirring was continued for $1 \mathrm{~h}$. After completion of the reaction as determined by TLC, $\mathrm{H}_{2} \mathrm{O}(10 \mathrm{~mL})$ was added and the organic layer was washed with further portions of $\mathrm{H}_{2} \mathrm{O}(2 \times 10 \mathrm{~mL})$ and brine $(10 \mathrm{~mL})$, then dried over $\mathrm{MgSO}_{4}$ and evaporated in vacuo. The residue was then triturated with cold $\mathrm{EtOH}$ and filtered to provide the title product $\mathbf{6}$, which was purified by re-crystallization from $\mathrm{EtOH}$.

\section{4af: methyl 1-oxo-2',4'-diphenyl-1,3-dihydrospiro[indene-2,3'-pyrrolidine]-5'- carboxylate}

Yield $0.34 \mathrm{~g}(88 \%)$; white needles, mp: $180^{\circ} \mathrm{C} \pm 2$, IR (KBr): $1681,1743,3447 \mathrm{~cm}^{-1},{ }^{1} \mathrm{H}$ NMR $\delta: 2.69-2.84(\mathrm{AB}, 2 \mathrm{H}, J=18 \mathrm{~Hz}, \mathrm{H}-3), 3.55$ (s, br, $1 \mathrm{H}, \mathrm{NH}), 3.79$ (s, 3H, OCH $), 3.90$ (d, 1H, $J=6 \mathrm{~Hz}, \mathrm{H}-5$ ') 4.32 (d, 1H, $J=6 \mathrm{~Hz}, \mathrm{H}-4$ ') 4.53 (s, 1H, H-2'), 6.94-7.35 (m, 14H,

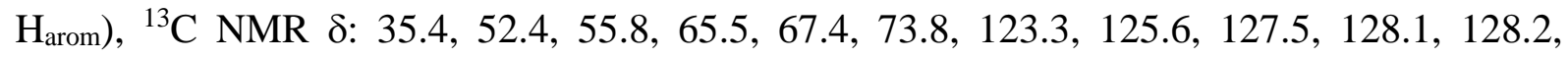
$129.5,130.1,131.8,133.3,134.9,134.9,135.8,139.0,142.0,170.2,207.2$, Anal. Calcd. for $\mathrm{C}_{26} \mathrm{H}_{23} \mathrm{NO}_{3}$ : C, 78.57, H, 5.83, N, 3.52, Found: C,78.56, H, 5.99, N, 3.49.

\section{4ag: methyl 1-oxo-2'-(p-methylphenyl)-4'-phenyl-1, 3-dihydrospiro[indene-2,3'- pyrrolidine]-5'-carboxylate}

Yield $0.31 \mathrm{~g}(77 \%)$; white crystals, mp: $200{ }^{\circ} \mathrm{C} \pm 2$, IR (KBr): $1683,1740,3449 \mathrm{~cm}^{-1},{ }^{1} \mathrm{H}$ NMR $\delta: 2.09$ (s, 3H, Ar- $\left.\mathrm{CH}_{3}\right), 2.68-2.82(\mathrm{AB}, 2 \mathrm{H}, J=18 \mathrm{~Hz}, \mathrm{H}-3), 3.52$ (s, br, 1H, NH), 3.73 (s, 3H, $\mathrm{OCH}_{3}$ ), 3.90 (d, 1H, $J=6 \mathrm{~Hz}, \mathrm{H}-5$ '), 4.31 (d, 1H , $J=6 \mathrm{~Hz}, \mathrm{H}-4$ '), 4.48 (s,1H, H-2'), 6.77-7.33 (m, 13H, $\left.\mathrm{H}_{\text {arom }}\right),{ }^{13} \mathrm{C} \mathrm{NMR} \delta: 21.3,35.2,51.4,55.8,65.5,67.4,72.8,113.3,119.4$, 125.6, 127.5, 128.1, 128.2, 129.5, 130.1 131.8, 133.3, 134.9, 134.9, 135.8, 139, 152, 170.28, 208.2, Anal. Calcd. for $\mathrm{C}_{27} \mathrm{H}_{25} \mathrm{NO}_{3}$ : C, 78.81, H, 6.12, N, 3.40, Found: C, 78.83, H, 6.12, N, 3.39 . 
4ah: methyl 1-oxo-2'-( $p$-methoxyphenyl)-4'-phenyl-1, 3-dihydrospiro[indene-2,3'pyrrolidine]-5'-carboxylate

Yield $0.31 \mathrm{~g}$ (74\%), white needles, mp: $192{ }^{\circ} \mathrm{C} \pm 2$, IR (KBr): $168,1740,3449 \mathrm{~cm}^{-1},{ }^{1} \mathrm{H}$ NMR $\delta: 2.66-2.81(\mathrm{AB}, 2 \mathrm{H}, J=18 \mathrm{~Hz}, \mathrm{H}-3), 3.53$ (s, br, 1H, NH), $3.58\left(\mathrm{~s}, 3 \mathrm{H}, \mathrm{Ar}_{-} \mathrm{OCH}_{3}\right)$, 3.74 (s, 3H, OCH 3 ), 3.90 (d, 1H, J = 6 Hz, H-5'), 4.33 (d, 1H, J = 6 Hz, H-4'), 4.49 (s, 1H, H$\left.2^{\prime}\right)$, 6.51-7.34 (m, 13H, $\left.\mathrm{H}_{\text {arom }}\right),{ }^{13} \mathrm{C}$ NMR $\delta: 35.5,52.4,52.5,65.6,67.4,73.8,123.3,126.6$, $127.5,128.1,128.1,129.5,130.1,132.8,133.3,134.9,134.9,135.8,139.1,152.0,173.2$, 208.2. Anal. Calcd. for $\mathrm{C}_{27} \mathrm{H}_{25} \mathrm{NO}_{4}$ : C, 75.86, H, 5.89, N, 3.28, Found: C, 75.83, H, 5.86, N, 3.29 .

4ai: methyl 1-oxo-2' -(p-chlorophenyl)-4'-phenyl-1,3-dihydrospiro[indene-2,3'pyrrolidine]-5'-carboxylate

Yield $0.31 \mathrm{~g}(74 \%)$, white crystals, mp: $162{ }^{\circ} \mathrm{C} \pm 2$, IR (KBr): $1689,1750,3449 \mathrm{~cm}^{-1},{ }^{1} \mathrm{H}$ NMR $\delta$ : 2.67-2.85 (AB, 2H, $J=18 \mathrm{~Hz}, \mathrm{H}-3), 3.23$ (s, br, 1H, NH), 3.77 (s, 3H, OCH $), 3.92$ (d, 1H, $J=6 \mathrm{~Hz}, \mathrm{H}-5^{\prime}$ '), 4.28 (d, 1H, $J=6 \mathrm{~Hz}, \mathrm{H}-4^{\prime}$ ), 4.47 (s, 1H, H-2'), 6.95-7.33 (m, 13H, $\left.\mathrm{H}_{\text {arom}}\right),{ }^{13} \mathrm{C}$ NMR $\delta: 35.4,52.4,55.8,65.6,67.4,73.8,123.3,125.6,127.5,128.1,128.2$, 129.4, 130.1, 132.8, 133.3, 134.9, 134.9, 135.8, 139.1, 152.06, 173.2, 208.2, Anal. Calcd. for $\mathrm{C}_{26} \mathrm{H}_{22} \mathrm{ClNO}_{3}$ : C, 72.30, H, 5.13, N, 3.28; Found: C, 72.32, H, 5.16, N, 3.29.

\section{4bf: methyl 1-oxo-2'-phenyl-4' -(p-methylphenyl)-1,3-dihydrospiro[indene-2,3'-} pyrrolidine]-5'-carboxylate

Yield $0.31 \mathrm{~g}(77 \%)$, white needles, mp: $210{ }^{\circ} \mathrm{C} \pm 2$, IR (KBr): $1689,1750,3449 \mathrm{~cm}^{-1},{ }^{1} \mathrm{H}$ NMR $\delta: 2.29\left(\mathrm{~s}, 3 \mathrm{H}, \mathrm{Ar}-\mathrm{CH}_{3}\right), 2.75-2.79(\mathrm{AB}, 2 \mathrm{H}, J=18 \mathrm{~Hz}, \mathrm{H}-3), 3.02$ (s, br, 1H, NH), 3.88 (s, 3H, $\mathrm{OCH}_{3}$ ), 3.89 (d, 1H, $J=6 \mathrm{~Hz}, \mathrm{H}-5$ '), 4.27 (d, 1H, $J=6 \mathrm{~Hz}, \mathrm{H}-4$ '), 4.49 (s, 1H, H-2'), 6.98-7.23 (m, 13H, $\left.\mathrm{H}_{\text {arom }}\right),{ }^{13} \mathrm{C}$ NMR $\delta: 21.0,35.4,52.4,55.8,65.6,67.4,73.8,123.3,125.5$, $127.5,128.3,128.2,129.5,130.2,132.9,133.3,134.9,134.9,135.8,139.1,152.0,173.2$, 208.2, Anal. Calcd. for $\mathrm{C}_{27} \mathrm{H}_{25} \mathrm{NO}_{3}$ : C, 78.81, H, 6.12, N, 3.40, Found: C, 78.82, H, 6.16, N, 3.42 .

4bg: methyl 1-oxo-2', 4'-di (p-methylphenyl)-1,3-dihydrospiro[indene-2,3'pyrrolidine]-5'-carboxylate

Yield $0.33 \mathrm{~g}(78 \%)$, white needles, mp: $180^{\circ} \mathrm{C} \pm 2$, IR $(\mathrm{KBr}): 1689,1750,3449 \mathrm{~cm}^{-1},{ }^{1} \mathrm{H}$ NMR $\delta: 2.06$ (s, 3H, Ar- $\left.\mathrm{CH}_{3}\right), 2.28$ (s, 3H, Ar-CH $), 2.68-2.83(\mathrm{AB}, 2 \mathrm{H}, J=18 \mathrm{~Hz}, \mathrm{H}-3)$, 3.13 (s, br, 1H, NH), 3.76 (s, 3H, $\left.\mathrm{OCH}_{3}\right), 3.85$ (d, 1H, $J=6 \mathrm{~Hz}, \mathrm{H}-5$ '), 4.26 (d, 1H, J = 6 Hz,

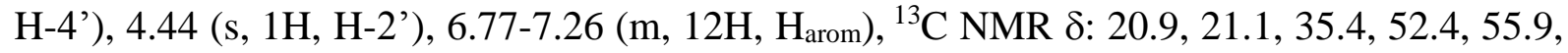
65.6, 67.6, 73.8, 123.3, 125.4, 127.5, 128.3, 128.2, 129.4, 130.2, 132.9, 133.3, 134.9, 134.9, 135.8, 139.1, 152.0, 173.2, 208.2. Anal. Calcd. for $\mathrm{C}_{28} \mathrm{H}_{27} \mathrm{NO}_{3}$ : C, 79.03, $\mathrm{H}, 6.40, \mathrm{~N}, 3.29$, Found: C, 79.05, H, 6.43, N, 3.30.

4bh: methyl 1-oxo-2' -(p-methoxyphenyl)-4' -(p-methylphenyl)-1,3-

dihydrospiro[indene-2,3'-pyrrolidine]-5'-carboxylate

Yield $0.35 \mathrm{~g}(80 \%)$, white needles, mp: $168^{\circ} \mathrm{C} \pm 2$, IR (KBr): $1680,1754,3450 \mathrm{~cm}^{-1},{ }^{1} \mathrm{H}$ NMR $\delta: 2.36\left(\mathrm{~s}, 3 \mathrm{H}, \mathrm{Ar}-\mathrm{CH}_{3}\right), 2.73-2.90(\mathrm{AB}, 2 \mathrm{H}, J=18 \mathrm{~Hz}, \mathrm{H}-3), 2.79$ (s, br, 1H, NH), 3.76 (s, 3H, Ar-OCH 3 ), 3.79 (s, 3H, OCH $\left.{ }_{3}\right), 3.93$ (d, 1H, $\left.J=6 \mathrm{~Hz}, \mathrm{H}-5^{\prime}\right), 4.36(\mathrm{~d}, 1 \mathrm{H}, J=6 \mathrm{~Hz}$,

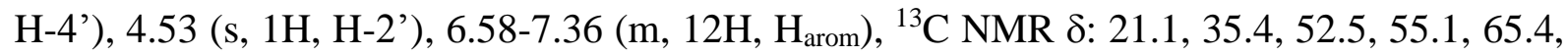
67.4, 73.4, 113.5, 123.3, 125.4, 127.5, 128.2, 129.2, 129.4, 130.5, 132.9, 133.5, 134.9, 134.9, 135.8, 139.1, 159.2, 173.3, 208.0 Anal. Calcd. for $\mathrm{C}_{28} \mathrm{H}_{27} \mathrm{NO}_{4}$ : C, 76.17, H, 6.16, N, 3.17, Found: C, 76.19, H, 6.13, N, 3.17. 
4bi: methyl 1-oxo-2'-(p-chlorophenyl)-4'-(p-methylphenyl)-1,3-dihydrospiro[indene2,3'-pyrrolidine]-5'-carboxylate

Yield $0.40 \mathrm{~g}(92 \%)$, yellow crystals, mp: $184^{\circ} \mathrm{C} \pm 2$, IR (KBr): $1682,1754,3449 \mathrm{~cm}^{-1},{ }^{1} \mathrm{H}$ NMR $\delta: 2.36$ (s, 3H, Ar-CH3), 2.76-2.91 (AB, 2H, $J=18 \mathrm{~Hz}, \mathrm{H}-3), 2.82$ (s, br, 1H, NH), 3.81 (s, 3H, $\mathrm{OCH}_{3}$ ), 3.94 (d, 1H, $J=6 \mathrm{~Hz}, \mathrm{H}-5$ '), 4.35 (d, 1H, $J=6 \mathrm{~Hz}, \mathrm{H}-4$ '), 4.52 (s, 1H, H-2'), 6.85-7.34 (m, $\left.12 \mathrm{H}, \mathrm{H}_{\text {arom }}\right),{ }^{13} \mathrm{C} \mathrm{NMR} \delta: 21.1,35.5,52.4,55.9,65.6,67.5,73.8,123.3,125.39$, $127.5,128.2,129.2,129.4,130.5,132.9,133.5,134.9,134.9,135.8,139.1,152.0,172.2$, 208.2 Anal. Calcd. for $\mathrm{C}_{27} \mathrm{H}_{24} \mathrm{ClNO}_{3}:$ C, 72.72, H, 5.42, N, 3.14, Found: C, 72.72, H, 5.42, N, 3.14 .

4cf: methyl 1-oxo-2'-phenyl-4' -(p-methoxyphenyl)-1,3-dihydrospiro[indene-2,3'pyrrolidine]-5'-carboxylate

Yield $0.27 \mathrm{~g}(65 \%)$, white needles, mp: $188{ }^{\circ} \mathrm{C} \pm 2$, IR (KBr): $1680,1754,3439 \mathrm{~cm}^{-1},{ }^{1} \mathrm{H}$ NMR $\delta: 2.69-2.85(\mathrm{AB}, 2 \mathrm{H}, J=18 \mathrm{~Hz}, \mathrm{H}-3), 3.16$ (s, br, 1H, NH), 3.73 (s, 3H, Ar-OCH ${ }_{3}$, $3.76\left(\mathrm{~s}, 3 \mathrm{H}, \mathrm{OCH}_{3}\right), 3.87$ (d, 1H, $J=6 \mathrm{~Hz}, \mathrm{H}-5$ '), 4.24 (d, 1H, $J=6 \mathrm{~Hz}, \mathrm{H}-4$ ') 4.46 (s, 1H, H2'), 6.81-7.23 (m, 13H, $\left.\mathrm{H}_{\text {arom }}\right),{ }^{13} \mathrm{C}$ NMR $\delta: 35.6,52.4,55.4,65.9,67.7,74,114.2,123.3$, 125.6, 127.5, 128.1, 128.2, 129.5, 130.1, 131.8, 133.3, 134.9, 134.9, 135.8, 139.0, 142, 158.7, 173.3, 208.0 Anal. Calcd. for $\mathrm{C}_{27} \mathrm{H}_{25} \mathrm{NO}_{4}$ : C, 75.86, H, 5.89, N, 3.28, Found: C, 75.84, H, $5.86, \mathrm{~N}, 3.28$.

\section{4cg: methyl 1-oxo-2'-(p-methylphenyl)-4'-(p-methoxyphenyl)-1,3-dihydrospiro} [indene-2,3'-pyrrolidine]-5'-carboxylate

Yield $0.28 \mathrm{~g}(65 \%)$, white crystals, mp: $142{ }^{\circ} \mathrm{C} \pm 2$, IR (KBr): $1680,1754,3439 \mathrm{~cm}^{-1},{ }^{1} \mathrm{H}$ NMR $\delta: 2.09$ (s, 3H, Ar- $\left.\mathrm{CH}_{3}\right), 2.68-2.87(\mathrm{AB}, 2 \mathrm{H}, J=18 \mathrm{~Hz}, \mathrm{H}-3), 2.74$ (s, br, 1H, NH), 3.69 (s, 3H, Ar-OCH $\left.{ }_{3}\right), 3.73$ (s, 3H, OCH 3$), 3.84(\mathrm{~d}, 1 \mathrm{H}, J=6 \mathrm{~Hz}, \mathrm{H}-5$ '), 4.25 (d, 1H, J = $6 \mathrm{~Hz}, \mathrm{H}-$

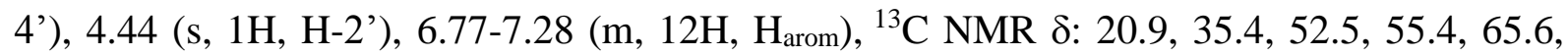
67.4, 73.6, 114.2, 125.3, 127.5, 128.2, 129.2, 129.4, 130.5, 132.9, 133.5, 134.9, 134.9, 135.8, 139.1, 158.7, 173.1, 208.0 Anal. Calcd. for $\mathrm{C}_{28} \mathrm{H}_{27} \mathrm{NO}_{4}$ : C, 76.17, H, 6.16, N, 3.17, Found: C, 76.19, H, 6.13, N, 3.16 .

\section{4ch: methyl 1-oxo-2',4'-di(p-methoxyphenyl)-1,3-dihydrospiro [indene-2,3'-}

\section{pyrrolidine]-5'-carboxylate}

Yield $0.29 \mathrm{~g}(64 \%)$, beige crystals, mp: $150{ }^{\circ} \mathrm{C} \pm 2$, IR (KBr): $1679,1754,3440 \mathrm{~cm}^{-1},{ }^{1} \mathrm{H}$ NMR $\delta: 2.67-2.84(\mathrm{AB}, 2 \mathrm{H}, J=18 \mathrm{~Hz}, \mathrm{H}-3), 2.78$ (s, br, 1H, NH), 3.58 (s, 3H, Ar-OCH $)$, 3.74 (s, 3H, Ar-OCH 3 ), 3.77 (s, 3H, $\left.\mathrm{OCH}_{3}\right), 3.83$ (d, 1H, $J=6 \mathrm{~Hz}, \mathrm{H}-5$ '), 4.25 (d, 1H, $J=6$

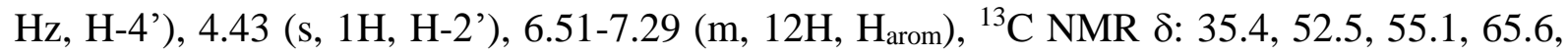
67.5, 73.5, 113.4, 123.3, 125.3, 127.5, 128.2, 129.2, 129.4, 130.5, 132.9, 133.5, 134.9, 134.9, 135.8, 139.1, 159.1, 173.1, 208.1. Anal. Calcd. for $\mathrm{C}_{28} \mathrm{H}_{27} \mathrm{NO}_{5}$ : C, 73.51, H, 5.95, N, 3.06, Found: C, 73.53, H, 5.94, N, 3.07.

4ci: methyl 1-oxo-2' -(p-chlorophenyl)-4'-(p-methoxyphenyl)-1, 3-

dihydrospiro[indene-2,3'-pyrrolidine]-5'-carboxylate

Yield $0.30 \mathrm{~g}(67 \%)$, white crystals, mp: $158^{\circ} \mathrm{C} \pm 2$, IR $(\mathrm{KBr}): 1677,1754,3449 \mathrm{~cm}^{-1},{ }^{1} \mathrm{H}$ NMR $\delta: 2.75-2.95(\mathrm{AB}, 2 \mathrm{H}, J=18 \mathrm{~Hz}, \mathrm{H}-3), 2.81(\mathrm{~s}, \mathrm{br}, 1 \mathrm{H}, \mathrm{NH}), 3.81\left(\mathrm{~s}, 3 \mathrm{H}, \mathrm{Ar}-\mathrm{OCH}_{3}\right)$, $3.89\left(\mathrm{~s}, 3 \mathrm{H}, \mathrm{OCH}_{3}\right), 3.95(\mathrm{~d}, 1 \mathrm{H}, J=6 \mathrm{~Hz}, \mathrm{H}-5$ ') 4.39 (d, $1 \mathrm{H}, J=6 \mathrm{~Hz}, \mathrm{H}-4$ ') 4.57 (s, 1H, H2'), 6.89-7.41 (m, 12H, $\left.\mathrm{H}_{\text {arom }}\right),{ }^{13} \mathrm{C} \mathrm{NMR} \delta: 35.4,52.5,55.0,65.5,67.5,73.5,114.3,123.3$, 125.3 ,127.5, 128.2, 129.2, 129.4, 130.5, 132.9, 133.5, 134.9, 134.9, 135.8, 139.1, 151.8, 173.1, 207.5, Anal. Calcd. for $\mathrm{C}_{27} \mathrm{H}_{24} \mathrm{ClNO}_{4}$ : C, 70.20, H, 5.24, N, 3.03, Found: C, 70.20, H, 5.26, N, 3.06. 
4df: methyl 1-oxo-2'-phenyl-4'-(p-chlorophenyl)-1,3-dihydrospiro[indene-2,3'pyrrolidine]-5'-carboxylate

Yield $0.29 \mathrm{~g}(68 \%)$, beige needles, mp: $200{ }^{\circ} \mathrm{C} \pm 2$, IR $(\mathrm{KBr}): 1677,1754,3449 \mathrm{~cm}^{-1},{ }^{1} \mathrm{H}$

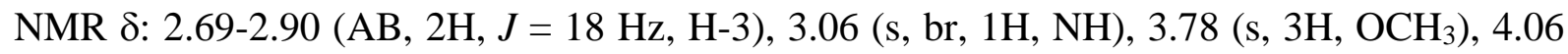
(d, 1H, $J=6 \mathrm{~Hz}, \mathrm{H}-5^{\prime}$ '), 4.28 (d, 1H, $J=6 \mathrm{~Hz}, \mathrm{H}-4$ ') 4.49 (s, 1H, H-2'), 6.98-8.25 (m, 13H, $\mathrm{H}_{\text {arom })},{ }^{13} \mathrm{C}$ NMR $\delta: 35.7,52.7,55.2,65.6,67.2,74.4,123.5,125.3,127.5,128.2,129.2,129.4$, $130.5,132.9,133.5,134.9,134.9,135.8,139.1,151.1,172.5,206.7$ Anal. Calcd. for $\mathrm{C}_{27} \mathrm{H}_{24} \mathrm{ClNO}_{4}$ : C, 70.20, H, 5.24, N, 3.03, Found: C, 70.25, H, 5.24, N, 3.03.

4dg: methyl 1-oxo-2'-(p-methylphenyl)-4'-(p-chlorophenyl)-1, 3-dihydrospiro[indene2,3'-pyrrolidine]-5'-carboxylate

Yield $0.28 \mathrm{~g}(63 \%)$, brown crystals, mp: $168{ }^{\circ} \mathrm{C} \pm 2$, IR (KBr) : $1677,1754,3449 \mathrm{~cm}^{-1},{ }^{1} \mathrm{H}$ NMR $\delta: 2.17$ (s, 3H, Ar- $\left.\mathrm{CH}_{3}\right), 2.77-2.93(\mathrm{AB}, 2 \mathrm{H}, J=18 \mathrm{~Hz}, \mathrm{H}-3), 2.83$ (s, br, 1H, NH), 3.86 (s, 3H, $\mathrm{OCH}_{3}$ ), 4.13 (d, 1H, $J=6 \mathrm{~Hz}, \mathrm{H}-5$ '), 4.38 (d, 1H, J = 6 Hz, H-4'), 4.55 (s, 1H, H-2'), 6.88-8.26 (m, 12H, $\left.\mathrm{H}_{\text {arom }}\right),{ }^{13} \mathrm{C}$ NMR $\delta: 20.9,35.6,52.7,55.3,65.5,66.9,74.1,123.5,125.3$ ,127.5, 128.2, 129.2, 129.4, 130.5, 132.9, 133.5, 134.9, 134.9, 135.8, 139.1, 151.2, 172.5, 206.9, Anal. Calcd. for $\mathrm{C}_{27} \mathrm{H}_{24} \mathrm{ClNO}_{3}$ : C, 72.72, H, 5.42, N, 3.14, Found : C, 72.66, H, 5.38, $\mathrm{N}, 3.15$.

4dh: methyl 1-oxo-2' -(p-methoxyphenyl)-4' -(p-chlorophenyl)-1,3dihydrospiro[indene-2,3'-pyrrolidine]-5'-carboxylate

Yield $0.31 \mathrm{~g}(69 \%)$, beige crystals, mp: $152{ }^{\circ} \mathrm{C} \pm 2$, IR (KBr): $1679,1759,3450 \mathrm{~cm}^{-1},{ }^{1} \mathrm{H}$ NMR $\delta: 2.75-2.90(\mathrm{AB}, 2 \mathrm{H}, J=18 \mathrm{~Hz}, \mathrm{H}-3), 3.17$ (s, br, 1H, NH), 3.66 (s, 3H, Ar-OCH $)_{3}$, 3.68 (s, 3H, $\left.\mathrm{OCH}_{3}\right), 4.13$ (d, 1H, J = 6 Hz, H-5'), 4.34 (d, 1H, J = 6 Hz, H-4'), 4.52 (s, 1H, H-

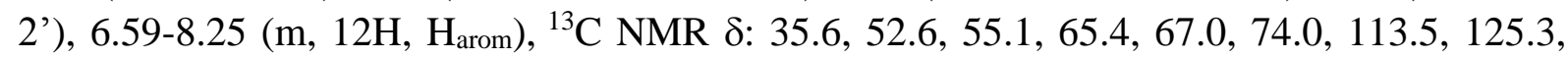
$127.5,128.3,129.2,129.5,130.5,132.9,133.5,134.9,134.9,135.8,139.2,159.2,172.6$, 207.0. Anal. Calcd. for $\mathrm{C}_{27} \mathrm{H}_{24} \mathrm{ClNO}_{4}$ : C, 70.20, H, 5.24, N, 3.03, Found: C, 69.85, H, 5.14, N, 2.94 .

4di: methyl 1-oxo-2', 4'-di(p-chlorophenyl)-1,3-dihydrospiro[indene-2,3'pyrrolidine]-5'-carboxylate

Yield $0.33 \mathrm{~g}(72 \%)$, beige crystals, mp: $178^{\circ} \mathrm{C} \pm 2$, IR (KBr): $1679,1759,3450 \mathrm{~cm}^{-1},{ }^{1} \mathrm{H}$ NMR $\delta: 2.71-2.84(\mathrm{AB}, 2 \mathrm{H}, J=18 \mathrm{~Hz}, \mathrm{H}-3), 2.78$ (s, br, 1H, NH), 3.77 (s, 3H, OCH $), 4.06$ (d, 1H, $J=6 \mathrm{~Hz}, \mathrm{H}-5$ ') 4.28 (d, 1H, $J=6 \mathrm{~Hz}, \mathrm{H}-4$ ') 4.46 (s, 1H, H-2'), 6.96-8.17 (m, 12H, $\mathrm{H}_{\text {arom })},{ }^{13} \mathrm{C} \mathrm{NMR} \delta: 35.8,52.7,54.9,65.5,66.8,73.6,123.6,125.3,127.5,128.2,129.2,129.4$, 130.5, 132.9, 133.5, 134.9, 134.9, 135.8, 139.1, 151.0, 172.5, 206.4 Anal. Calcd. for $\mathrm{C}_{26} \mathrm{H}_{21} \mathrm{Cl}_{2} \mathrm{NO}_{3}$ : C, 66.96, H, 4.54, N, 3.00, Found: C, 66.78, H, 4.42, N, 2.87.

4ef: methyl 1-oxo-2'-phenyl-4' -(p-nitrophenyl)-1,3-dihydrospiro[indene-2,3'pyrrolidine]-5'-carboxylate

Yield $0.39 \mathrm{~g}(90 \%)$, white needles, $\mathrm{mp}: 210^{\circ} \mathrm{C} \pm 2$, IR (KBr): 1676, 1759, $3449 \mathrm{~cm}^{-1}$, ${ }^{1} \mathrm{H}$ NMR $\delta: 2.70-2.82(\mathrm{AB}, 2 \mathrm{H}, J=18 \mathrm{~Hz}, \mathrm{H}-3), 2.92(\mathrm{~s}, \mathrm{br}, 1 \mathrm{H}, \mathrm{NH}), 3.77\left(\mathrm{~s}, 3 \mathrm{H}, \mathrm{OCH}_{3}\right)$, 3.90 (d, 1H, $J=6 \mathrm{~Hz}, \mathrm{H}-5$ '), 4.23 (d, 1H, $J=6 \mathrm{~Hz}, \mathrm{H}-4$ '), 4.45 (s, 1H, H-2'), 6.96-7.50 (m,

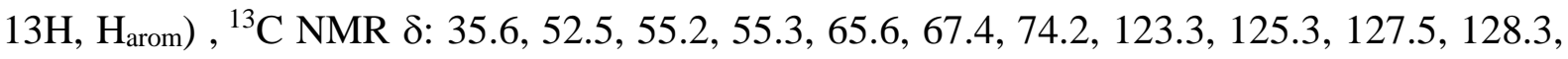
129.2, 129.5, 130.5, 132.9, 133.5, 134.9, 134.9, 135.8, 139.2, 151.6, 172.9, 207.5 Anal. Calcd. for $\mathrm{C}_{26} \mathrm{H}_{22} \mathrm{~N}_{2} \mathrm{O}_{5}$ : C, 72.30, H, 5.13, N, 3.24, Found : C, 72.32, H, 5.11, N, 3.24. 
4eg: methyl 1-oxo-2'-(p-methylphenyl)-4'-(p-nitrophenyl)-1, 3-dihydrospiro [indene2,3'-pyrrolidine]-5'-carboxylate

Yield $0.41 \mathrm{~g}$ (92\%), white crystals, mp: $160{ }^{\circ} \mathrm{C} \pm 2$, IR (KBr): 1676, 1759, $3449 \mathrm{~cm}^{-1}$, ${ }^{1} \mathrm{H}$ NMR $\delta: 2.11$ (s, 3H, Ar-CH3), 2.69-2.90 (AB, 2H, $\left.J=18 \mathrm{~Hz}, \mathrm{H}-3\right), 3.06$ (s, br, 1H, NH), $3.63\left(\mathrm{~s}, 3 \mathrm{H}, \mathrm{OCH}_{3}\right), 4.26$ (d, $1 \mathrm{H}, J=6 \mathrm{~Hz}, \mathrm{H}-5$ '), 4.31 (d, 1H, $J=6 \mathrm{~Hz}, \mathrm{H}-4$ ') 4.54 (s, 1H, H$\left.2^{\prime}\right)$, 6.71-7.57 (m, 12H, Harom), ${ }^{13} \mathrm{C}$ NMR $\delta: 21.0,35.7,52.5,55.2,64.2,67.2,70.3,113.6$, $123.3,125.3,127.5,128.3,129.2,129.5,130.5,132.9,133.5,134.9,134.9,135.8,139.2$, 142.2, 172.5, 198.6 Anal. Calcd. for $\mathrm{C}_{27} \mathrm{H}_{24} \mathrm{~N}_{2} \mathrm{O}_{5}$ : C, 71.04, H, 5.30, N, 6.14, Found : C, 70.98., H, 5.28, N, 6.13.

4eh: methyl 1-oxo-2'-(p-methoxyphenyl)-4' -(p-nitrophenyl)-1,3-dihydrospiro[indene2,3'-pyrrolidine]-5'-carboxylate

Yield $0.28 \mathrm{~g}(61 \%)$, white needles, mp: $120{ }^{\circ} \mathrm{C} \pm 2$, IR (KBr) : $1679,1760,3448 \mathrm{~cm}^{-1},{ }^{1} \mathrm{H}$ NMR $\delta: 2.68-2.80(\mathrm{AB}, 2 \mathrm{H}, J=18 \mathrm{~Hz}, \mathrm{H}-3), 3.07$ (s, br, 1H, NH), 3.78 (s, 3H, Ar-OCH 3 ), $3.81\left(\mathrm{~s}, 3 \mathrm{H}, \mathrm{OCH}_{3}\right), 3.85$ (d, $1 \mathrm{H}, J=6 \mathrm{~Hz}, \mathrm{H}-5$ '), 4.27 (d, 1H, $J=6 \mathrm{~Hz}, \mathrm{H}-4$ '), 4.45 (s, 1H, H$\left.2^{\prime}\right)$, 6.52-7.7 (m, 12H, $\left.\mathrm{H}_{\text {arom}}\right),{ }^{13} \mathrm{C} \mathrm{NMR} \delta: 35.4,52.6,55.1,65.3,67.1,73.5,113.5,125.3$ ,127.5, 128.2, 129.2, 129.4, 130.5, 132.9, 133.5, 134.9, 134.9, 135.8, 139.1, 139.4, 172.8, 207.7, Anal. Calcd. for $\mathrm{C}_{27} \mathrm{H}_{24} \mathrm{~N}_{2} \mathrm{O}_{6}$ : C,68.63, H, 5.12, N, 5.93, Found : C, 68.61, H, 5.02, N, 5.87 .

4ei: methyl 1-oxo-2'-(p-chlorophenyl)-4'-(p-nitrophenyl)-1,3-dihydrospiro[indene2,3'-pyrrolidine]-5'-carboxylate

Yield $0.29 \mathrm{~g}(61 \%)$, white crystals, mp: $130{ }^{\circ} \mathrm{C} \pm 2$, IR (KBr): $1680,1760,3449 \mathrm{~cm}^{-1},{ }^{1} \mathrm{H}$ NMR $\delta:$ 2.65-2.83 (AB, 2H, $J=18 \mathrm{~Hz}, \mathrm{H}-3), 2.64$ (s, br, 1H, NH), 3.73 (s, 3H, OCH $), 3.90$ (d, 1H, $J=6 \mathrm{~Hz}, \mathrm{H}-5$ ') 4.23 (d, 1H, $J=6 \mathrm{~Hz}, \mathrm{H}-4$ '), 4.43 (s, 1H, H-2'), 6.95-7.85 (m, 12H,

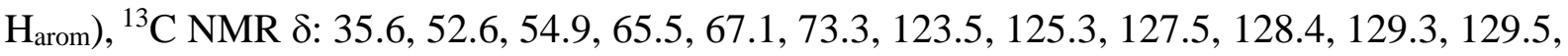
130.5, 132.9, 133.5, 134.9, 134.9, 135.8, 139.6, 142.2, 151.4, 172.9, 207.1, Anal. Calcd. for $\mathrm{C}_{26} \mathrm{H}_{21} \mathrm{ClN}_{2} \mathrm{O}_{5}: \mathrm{C}, 65.48, \mathrm{H}, 4.44, \mathrm{~N}, 5.87$, Found : C, 65.56, H, 4.52, N, 5.83.

5af: methyl 1-oxo-2', 4'-diphenyl-3, 4-dihydro-1H-spiro [naphthalene-2,3'pyrrolidine]-5'-carboxylate

Yield $0.33 \mathrm{~g}(82 \%)$; white crystals, mp: $168^{\circ} \mathrm{C} \pm 2$, IR $(\mathrm{KBr}): 1680,1743,3447 \mathrm{~cm}^{-1}$, ${ }^{1} \mathrm{H}$ NMR: 1.63 (td, $\left.J=5 \mathrm{~Hz}, 12.5 \mathrm{~Hz}, 1 \mathrm{H}, \mathrm{H}-3\right), 1.87$ (dt, $\left.J=3 \mathrm{~Hz}, 10.5 \mathrm{~Hz}, 1 \mathrm{H}, \mathrm{H}-4\right), 2.64$ (dt, $J=3 \mathrm{~Hz}, 10.5 \mathrm{~Hz}, 1 \mathrm{H}, \mathrm{H}-4), 2.69$ (s, br, 1H, NH), 3.01 (td, $J=5 \mathrm{~Hz}, 12.5 \mathrm{~Hz}, 1 \mathrm{H}, \mathrm{H}-3$ ), 3.63 (s, 3H, $\left.\mathrm{OCH}_{3}\right) ; 4.31(\mathrm{~d}, J=8.7 \mathrm{~Hz}, 1 \mathrm{H}, \mathrm{H}-5$ '), 4.44 (d, J=8.7 Hz, 1H, H-4'), 4.55 (s, 1H, $\mathrm{H}-2$ '), 6.8-7.53 (m, 14H, $\mathrm{H}_{\text {arom}}$ ), ${ }^{13} \mathrm{C} \mathrm{NMR}: 25.3,3.5,52.4,55.5,61.3,64.2,70.9,126.3$, 127.1, 127.5, 127.9, 128.0, 128.3, 129.3, 132.8, 133.0, 138.1, 139.1, 142.0, 173.8, 198.5 Anal. Calcd. for $\mathrm{C}_{27} \mathrm{H}_{25} \mathrm{NO}_{3}$ : C, 78.81, H, 6.12, N, 3.4. Found: C, 78.83, H, 6.2, N, 3.38.

5ag: methyl 1-oxo-2' -( $(p$-methylphenyl)-4'-phenyl-3,4-dihydro-1H-spiro[naphthalene2,3'-pyrrolidine]-5'-carboxylate

Yield $0.31 \mathrm{~g}(74 \%)$; white crystals $\mathrm{mp}: 158^{\circ} \mathrm{C} \pm 2$, IR $(\mathrm{KBr}): 1681,1740,3447 \mathrm{~cm}^{-1}$, ${ }^{1} \mathrm{H}$ NMR: 1.83 (td, $J=5 \mathrm{~Hz}, 12.5 \mathrm{~Hz}, 2 \mathrm{H}, \mathrm{H}-3$ ), 1.86 (dt, $\left.J=3 \mathrm{~Hz}, 10.5 \mathrm{~Hz}, 1 \mathrm{H}, \mathrm{H}-4\right), 2.09$ (s, $\left.3 \mathrm{H}, \mathrm{Ar}-\mathrm{CH}_{3}\right), 2.65$ (dt, $\left.J=3 \mathrm{~Hz}, 10.5 \mathrm{~Hz}, 1 \mathrm{H}, \mathrm{H}-4\right), 2.81$ (s, br, $\left.1 \mathrm{H}, \mathrm{NH}\right), 3.02$ (td, $J=5 \mathrm{~Hz}$, $12.5 \mathrm{~Hz}, 1 \mathrm{H}, \mathrm{H}-3), 3.67$ (s, 3H, CH 3 ), 4.28 (d, $J=8.7 \mathrm{~Hz}, 1 \mathrm{H}, \mathrm{H}-5$ '), 4.43 (d, $J=8.7 \mathrm{~Hz}, 1 \mathrm{H}$, H-4'), 4.53 (s, 1H, H-2'), 6.77-7.58 (m, 13H, Harom). ${ }^{13} \mathrm{C}$ NMR: 21.025 .3 , 30.4 , 52.4 , 55.6 , 61.3 , 64.2 , 70.7,126.3 ,127.0, 127.5, 127.8, 128.0, 128.3, 128.7, 129.3, 132.9, 132.0, 137.5, 138.2,142.1, 142.1, 173.9, 198.65. Anal. Calcd. for $\mathrm{C}_{28} \mathrm{H}_{27} \mathrm{NO}_{3}$ : C, 79.03, H, 6.4, N, 3.29. Found: C, 79.01, H, 6.2, N, 3.28. 
5ah: methyl 1-oxo-2'-(p-methoxyphenyl)-4'-phenyl-3, 4-dihydro-1H-spiro [naphthalene-2, 3'-pyrrolidine]-5'-carboxylate

Yield $0.22 \mathrm{~g}(52 \%)$; beige crystals, mp: $168^{\circ} \mathrm{C} \pm 2, \mathrm{IR}(\mathrm{KBr}): 1681,1741,3449 \mathrm{~cm}^{-1} ;{ }^{1} \mathrm{H}$ NMR: 1.65 (td, $J=5 \mathrm{~Hz}, 12.8 \mathrm{~Hz}, 1 \mathrm{H}, \mathrm{H}-3), 1.79$ (dt, $J=3 \mathrm{~Hz}, 11.5 \mathrm{~Hz}, 1 \mathrm{H}, \mathrm{H}-4), 2.66$ (dt, $J=3 \mathrm{~Hz}, 11.5 \mathrm{~Hz}, 1 \mathrm{H}, \mathrm{H}-4), 2.74$ (s, br, 1H, NH), 2.98 (td, $J=5 \mathrm{~Hz}, 12.8 \mathrm{~Hz}, 1 \mathrm{H}, \mathrm{H}-3), 3.59$ (s, 3H, $\left.\mathrm{OCH}_{3}\right), 3.65$ (s, 3H, Ar-OCH $), 4.29$ (d, J=8.7 Hz, 1H, H-5'), 4.42 (d, J = 8.7 Hz, 1H, H-4'), 4.49 (s, 1H, H-2'), 6.5-7.2 (m, 13H, $\mathrm{H}_{\text {arom }}$ ), ${ }^{13} \mathrm{C}$ NMR : 25.3, 30.4, 52.4, 55.1, 55.5, 61.0, 64.2, 113.3, 126.3, 127.1, 127.5, 128.0, 128.3, 129.1, 129.3, 132.8,133.0, 138.2, 142.1, 173.8, 198.7.Anal.Calcd. for $\mathrm{C}_{28} \mathrm{H}_{27} \mathrm{NO}_{4}$ : C, 76.17, H, 6.16, N, 3.17. Found: C, 76.18, H, 6.1 N, 3.28.

\section{5ai: methyl 1-oxo-2' -(p-chlorophenyl)-4'-phenyl-3, 4-dihydro-1H-spiro [naphthalene-} 2,3'-pyrrolidine]-5'-carboxylate

Yield $0.19 \mathrm{~g}(43 \%)$; white crystals, mp: $180^{\circ} \mathrm{C} \pm 2$, IR (KBr): $1679,1735,3446 \mathrm{~cm}^{-1} ;{ }^{1} \mathrm{H}$ NMR: 1.72 (td, $J=5 \mathrm{~Hz}, 12.8 \mathrm{~Hz}, 1 \mathrm{H}, \mathrm{H}-3), 1.95$ (dt, $J=3 \mathrm{~Hz}, 11 \mathrm{~Hz}, 1 \mathrm{H}, \mathrm{H}-4), 2.76$ (dt, $J=3 \mathrm{~Hz}, 11 \mathrm{~Hz}, 1 \mathrm{H}, \mathrm{H}-4), 2.81$ (s, br, $1 \mathrm{H}, \mathrm{NH}), 3.15$ (td, $J=5 \mathrm{~Hz}, 12.8 \mathrm{~Hz}, 1 \mathrm{H}, \mathrm{H}-3$ ), 3.77 (s, $3 \mathrm{H}, \mathrm{OCH}_{3}$ ), 4.40 (d, $J=8.7 \mathrm{~Hz}, 1 \mathrm{H}, \mathrm{H}-5$ ') 4.53 (d, $J=8.7 \mathrm{~Hz}, 1 \mathrm{H}, \mathrm{H}-4$ '), 4.65 (s, 1H, H-2'), 7.04-7.67 (m, 13H, $\mathrm{H}_{\text {arom }) .}{ }^{13} \mathrm{C} \mathrm{NMR}: 25.3,30.1,52.5,54.9,61.2,63.9,69.6,126.6,127.2$, $127.6,128.1,128.3,129.33,133.3,137.7,141.9,141.9,173.8,198.1$. Anal. Calcd. for $\mathrm{C}_{27} \mathrm{H}_{24} \mathrm{ClNO}_{3}$ : C, 27.72, H, 5.42, N, 3.14. Found: C, 27.62; H, 5.41, N, 3.12.

\section{5bf: methyl 1-oxo-2'-phenyl-4'-(p-methylphenyl)-3, 4-dihydro-1H-spiro}

[naphthalene-2,3'-pyrrolidine]-5'-carboxylate

Yield $0.27 \mathrm{~g}(65 \%)$, white crystals, mp: $138^{\circ} \mathrm{C} \pm 2$, IR (KBr): $1683,1735,3447 \mathrm{~cm}^{-1},{ }^{1} \mathrm{H}$ NMR: 1.68 (td, $J=5 \mathrm{~Hz}, 12.8 \mathrm{~Hz}, 1 \mathrm{H}, \mathrm{H}-3), 1.87$ (dt, $J=3 \mathrm{~Hz}, 11.5 \mathrm{~Hz}, 1 \mathrm{H}, \mathrm{H}-4), 2.20$ (s, $\left.3 \mathrm{H}, \mathrm{Ar}-\mathrm{CH}_{3}\right), 2.62$ (dt, $\left.J=3 \mathrm{~Hz}, 11.5 \mathrm{~Hz}, 1 \mathrm{H}, \mathrm{H}-4\right), 2.83$ (s, br, $\left.1 \mathrm{H}, \mathrm{NH}\right), 3.05$ (td, $J=5 \mathrm{~Hz}$, $12,8 \mathrm{~Hz}, 1 \mathrm{H}, \mathrm{H}-3), 3.69$ (s, 3H, CH 3 ), 4.27 (d, $J=8.7 \mathrm{~Hz}, 1 \mathrm{H}, \mathrm{H}-5$ '), 4.41 (d, $J=8.7 \mathrm{~Hz}, 1 \mathrm{H}$,

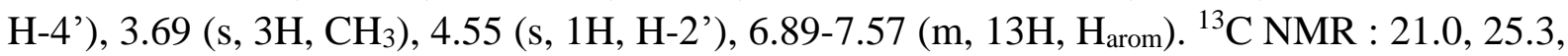
30.4, 52.4, 55.2, 61.2, 64.3,70.9, 126.3, 127.5, 127.8, 128.0, 128.0, 129.0, 129.1, 132.9, 136.7, 142.1, 173.9, 198.6. Anal. Calcd. for $\mathrm{C}_{28} \mathrm{H}_{27} \mathrm{NO}_{3}$ : C, 79.03, H, 6.4, N, 3.29. Found: C, 79.01, H, 6.2, N, 3.28.

\section{5bg: methyl 1-oxo-2',4'-di(p-methylphenyl)-3,4-dihydro-1H-spiro[naphthalene-2,3'- pyrrolidine]-5'-carboxylate}

Yield $0.32 \mathrm{~g}(73 \%)$; beige crystals, $\mathrm{mp}: 146^{\circ} \mathrm{C} \pm 2, \mathrm{IR}(\mathrm{KBr}): 1684,1749,3449 \mathrm{~cm}^{-1},{ }^{1} \mathrm{H}$ NMR : 1.65 (td, $J=4.5 \mathrm{~Hz}, 12.8 \mathrm{~Hz}, 1 \mathrm{H}, \mathrm{H}-3), 1.84$ (dt, $J=3 \mathrm{~Hz}, 10.5 \mathrm{~Hz}, 1 \mathrm{H}, \mathrm{H}-4), 2.01$ (s, $\left.6 \mathrm{H}, 2 \mathrm{Ar}-\mathrm{CH}_{3}\right), 2.65$ (dt, $\left.J=3 \mathrm{~Hz}, 10.5 \mathrm{~Hz}, 1 \mathrm{H}, \mathrm{H}-4\right), 2.69$ (s, br, 1H, NH), 2.94 (td, $J=4.5$ $\mathrm{Hz}, 12.8 \mathrm{~Hz}, 1 \mathrm{H}, \mathrm{H}-3), 3.70$ (s, 3H, $\mathrm{OCH}_{3}$ ), 4.32 (d, $J=8.4 \mathrm{~Hz}, 1 \mathrm{H}, \mathrm{H}-5$ '), 4.36 (d, $J=8.4$ Hz, 1H, H-4'), 4.49 (s, 1H, H-2'), 6.80-7.61 (m, 12H, Harom). ${ }^{13} \mathrm{C}$ NMR: 21.0, 21.0, 25.4, 52.4, 55.3, 61.2, 64.3, 70.7, 126.2, 127.5, 127.8, 128.0, 128.6, 129.0, 129.1, 132.9, 135.1, 136.0, 136.6, 137.4, 142.2, 173.9, 198.7, 207.1. Anal. Calcd. for $\mathrm{C}_{29} \mathrm{H}_{29} \mathrm{NO}_{3}: \mathrm{C}, 79.24, \mathrm{H}, 6.65, \mathrm{~N}$, 3.19. Found: C, 79.23, H, 6.66, N, 3.15.

5bh: methyl 1-oxo-2'-(p-methoxyphenyl)-4'-(p-methylphenyl)-3, 4-dihydro-1H-spiro [naphthalene-2,3'-pyrrolidine]-5'-carboxylate

Yield $0.29 \mathrm{~g}(64 \%)$; beige crystals, mp: $138^{\circ} \mathrm{C} \pm 2$, IR $(\mathrm{KBr}): 1682,1745,3449 \mathrm{~cm}^{-1},{ }^{1} \mathrm{H}$ NMR : $1.68(\mathrm{td}, J=5 \mathrm{~Hz}, 12.5 \mathrm{~Hz}, 1 \mathrm{H}, \mathrm{H}-3), 1.82(\mathrm{dt}, 5.1, J=3.5 \mathrm{~Hz}, 11.5 \mathrm{~Hz}, 1 \mathrm{H}, \mathrm{H}-4)$, 2.23 (s, 3H,Ar- $\mathrm{CH}_{3}$ ), 2.61 (dt, $\left.J=3.5 \mathrm{~Hz}, 11.5 \mathrm{~Hz}, 1 \mathrm{H}, \mathrm{H}-4\right), 2.77$ (s, br, 1H, NH), 3.03 (td, $J$ $=5 \mathrm{~Hz}, 12.5 \mathrm{~Hz}, 1 \mathrm{H}, \mathrm{H}-3), 3.59\left(\mathrm{~s}, 3 \mathrm{H}, \mathrm{OCH}_{3}\right), 3.37\left(\mathrm{~s}, 3 \mathrm{H}, \mathrm{Ar}-\mathrm{OCH}_{3}\right), 4.23(\mathrm{~d}, J=9 \mathrm{~Hz}, 1 \mathrm{H}$, $\mathrm{H}^{-5}$ '), 4.37 (d, $\left.J=9 \mathrm{~Hz}, 1 \mathrm{H}, \mathrm{H}-4^{\prime}\right), 4.50$ (s, 1H, H-2'), 6.50-7.59 (m, 12H, $\mathrm{H}_{\text {arom }) .}{ }^{13} \mathrm{C}$ NMR : 
21.0, 25.4, 30.4, 52.4, 55.3, 61.0, 64.2, 70.4, 113.3, 126.3, 127.5, 128, 129, 129.1, 129.1, 131, 132.9, 132.9, 135.1, 136.6, 142.2, 159, 173.8, 198.8. Anal. Calcd. for $\mathrm{C}_{29} \mathrm{H}_{29} \mathrm{NO}_{4}$ : C, 76.46, H, 6.42, N, 3.07. Found: C, 76.44, H, 6.39, N, 3.07.

\section{5bi: methyl 1-oxo-2'-(p-chlorophenyl)-4' -( $p$-methylphenyl)-3,4-dihydro-1H-spiro} [naphthalene-2,3'-pyrrolidine]-5'-carboxylate

Yield $0.43 \mathrm{~g}$ (94\%); yellow crystals, mp: $184^{\circ} \mathrm{C} \pm 2$, IR (KBr):1680,1743, $3446 \mathrm{~cm}^{-1},{ }^{1} \mathrm{H}$ NMR : 1.73 (td, $J=4.5 \mathrm{~Hz}, 12.5 \mathrm{~Hz}, 1 \mathrm{H}, \mathrm{H}-3), 1.93$ (dt, $J=3 \mathrm{~Hz}, 10.5 \mathrm{~Hz}, 1 \mathrm{H}, \mathrm{H}-4), 2.30$ (s, $\left.3 \mathrm{H}, \mathrm{Ar}_{-} \mathrm{CH}_{3}\right), 2.75$ (dt, $\left.J=3 \mathrm{~Hz}, 10.5 \mathrm{~Hz}, 1 \mathrm{H}, \mathrm{H}-4\right), 2.87$ (s, br, $\left.1 \mathrm{H}, \mathrm{NH}\right), 3.09$ (td, $J=4.5 \mathrm{~Hz}$, $12.5 \mathrm{~Hz}, 1 \mathrm{H}, \mathrm{H}-3), 3.74\left(\mathrm{~s}, 3 \mathrm{H}, \mathrm{OCH}_{3}\right), 4.35$ (d, $J=8.7 \mathrm{~Hz}, 1 \mathrm{H}, \mathrm{H}-5$ '), 4.48 (d, $J=8.7 \mathrm{~Hz}$, 1H, H-4'), 4.61 (s, 1H, H-2'), 6.97-7.63 (m, 12H, Harom). ${ }^{13} \mathrm{C}$ NMR : 21, 25.3, 30.1, 52.4, 54.6, 61.3, 64, 69.7, 126.5, 127.6, 128.1, 128.1, 129, 129.2, 129.3, 132.8, 133.2, 133.5, 134.6, 136.8, 138,142, 173.8, 198.1. Anal.Calcd. for $\mathrm{C}_{28} \mathrm{H}_{26} \mathrm{ClNO}_{3}: \mathrm{C}, 73.1, \mathrm{H}, 5.7, \mathrm{~N}, 3.05$. Found: C, 73.09, H, 5.5, N, 3.03.

\section{5cf: methyl 1-oxo-2'-phenyl-4' -(p-methoxyphenyl)-3,4-dihydro-1H-}

spiro[naphthalene-2,3'-pyrrolidine]-5'-carboxylate

Yield $0.30 \mathrm{~g}(69 \%)$; beige crystals, mp: $152^{\circ} \mathrm{C} \pm 2$, IR $(\mathrm{KBr}): 1680,1743,3449 \mathrm{~cm}^{-1},{ }^{1} \mathrm{H}$ NMR : 1.63 (td, $J=5 \mathrm{~Hz}, 13.8 \mathrm{~Hz}, 1 \mathrm{H}, \mathrm{H}-3), 1.85$ (dt, $J=3 \mathrm{~Hz}, 12.5 \mathrm{~Hz}, 1 \mathrm{H}, \mathrm{H}-4), 2.09$ (dt, $J$ $=3 \mathrm{~Hz}, 12.5 \mathrm{~Hz}, 1 \mathrm{H}, \mathrm{H}-4), 2.49$ (s, br, 1H, NH), 3.01 (td, $J=5 \mathrm{~Hz}, 13.8 \mathrm{~Hz}, 1 \mathrm{H}, \mathrm{H}-3), 3.70$ (s, 6H, 2Ar- $\left.\mathrm{OCH}_{3}\right), 4.31$ (d, $\left.J=8.7 \mathrm{~Hz}, 1 \mathrm{H}, \mathrm{H}-5^{\prime}\right), 4.39$ (d, $\left.J=8.7 \mathrm{~Hz}, 1 \mathrm{H}, \mathrm{H}-4^{\prime}\right), 4.57$ (s, 1H, $\left.\mathrm{H}-2^{\prime}\right), 6.76-7.52\left(\mathrm{~m}, 13 \mathrm{H}, \mathrm{H}_{\text {arom }}\right) .{ }^{13} \mathrm{C} \mathrm{NMR}: 30.1,31,52.5,54.6,55.2,61.1,66.4,170.4$, 113.7, 126.4, 127.5, 127.9, 128, 129.7, 130.3, 132.8, 133.1, 142.1, 158.6, 172.6, 198.5. Anal. Calcd. for $\mathrm{C}_{28} \mathrm{H}_{27} \mathrm{NO}_{4}$ : C, 76.17, H, 6.16, N, 3.17. Found: C, 76.18; H, 6.1; N, 3.28.

\section{5cg: methyl 1-oxo-2' -(p-methylphenyl)-4'-(p-methoxyphenyl)-3,4-dihydro-1H-spiro} [naphthalene-2,3'-pyrrolidine]-5'-carboxylate

Yield $0.26 \mathrm{~g}(59 \%)$; white crystals, $\mathrm{mp}: 140^{\circ} \mathrm{C} \pm 2$, IR (KBr): $1680,1743,3449 \mathrm{~cm}^{-1} ; 1 \mathrm{H}$ NMR: 1.65 (td, $J=5 \mathrm{~Hz}, 13.5 \mathrm{~Hz}, 1 \mathrm{H}, \mathrm{H}-3), 1.83$ (dt, $J=3 \mathrm{~Hz}, 12.5 \mathrm{~Hz}, 1 \mathrm{H}, \mathrm{H}-4), 2.81$ (dt, $J=3 \mathrm{~Hz}, 12.5 \mathrm{~Hz}, 1 \mathrm{H}, \mathrm{H}-4), 2.99$ (s, br, 1H, NH), 3.39 (td, $J=5 \mathrm{~Hz}, 13.5 \mathrm{~Hz}, 1 \mathrm{H}, \mathrm{H}-3$ ), 3.62 (s, 3H, Ar- $\left.\mathrm{CH}_{3}\right), 3.68$ (s, 3H, CH 3 ), 4.20 (d, $J=8.7 \mathrm{~Hz}, 1 \mathrm{H}, \mathrm{H}-5$ '), 4.37 (d, $J=8.7 \mathrm{~Hz}, 1 \mathrm{H}$, H-4'), 4.49 (s, 1H, H-2'), 6.5-7.57 (m, 12H, Harom). ${ }^{13} \mathrm{C}$ NMR: 15.3, 20.9, 35.5, 52.5, 55.3, 65.5, 65.9, 67.3, 123.4, 125.5, 126.6, 127.2, 128.7, 129, 130.1, 133, 133.1, 134.6, 135.9, 137.6, 139.5, 151.7, 173.0, 207.6. Anal. Calcd. for $\mathrm{C}_{29} \mathrm{H}_{29} \mathrm{NO}_{4}$ : C, 76.46, H, 6.42, N, 3.07. Found: C, 76.44, H, 6.39, N, 3.07.

5ch: methyl 1-oxo-2', 4'-di ( $p$-methoxyphenyl)-3, 4-dihydro-1H-spiro [naphthalene2,3'-pyrrolidine]-5'-carboxylate

Yield $0.28 \mathrm{~g}(61 \%)$; yellow crystals, $\mathrm{mp}: 151^{\circ} \mathrm{C} \pm 2$, IR $(\mathrm{KBr}): 1681,1745,3397 \mathrm{~cm}^{-1},{ }^{1} \mathrm{H}$ NMR : 1.15 (td, $J=5 \mathrm{~Hz}, 13.5 \mathrm{~Hz}, 1 \mathrm{H}, \mathrm{H}-3), 1.33$ (dt, $J=3 \mathrm{~Hz}, 12 \mathrm{~Hz}, 1 \mathrm{H}, \mathrm{H}-4), 2.07$ (s, 6H, $2 \mathrm{Ar}^{-\mathrm{OCH}_{3}}$ ), 2.97 (s, br, 1H, NH), $3.02(\mathrm{dt}, J=3 \mathrm{~Hz}, 12 \mathrm{~Hz}, 1 \mathrm{H}, \mathrm{H}-4), 3.43$ (td, $5 \mathrm{~Hz}, 13.5$ Hz, 1H, H-3), 3.76 (s, 3H, $\mathrm{OCH}_{3}$ ), 4.21 (d, $J=9 \mathrm{~Hz}, 1 \mathrm{H}, \mathrm{H}-5$ '), 4.23 (d, $J=9 \mathrm{~Hz}, 1 \mathrm{H}, \mathrm{H}-4$ '), 4.41 (s, $1 \mathrm{H}, \mathrm{H}-2$ '), $6.77-7.28$ (m, 12H, $\left.\mathrm{H}_{\text {arom }}\right){ }^{13} \mathrm{C} \mathrm{NMR}: 25.3,26.9,30.4,52.5,55.1,61.8$, 70.2, 113.3, 113.6, 126.3, 127.5, 128, 129, 130, 130.2, 131.4, 132.9, 132.9, 142.1, 174, 198.7. Anal. Calcd. for $\mathrm{C}_{29} \mathrm{H}_{29} \mathrm{NO}_{3}$ : C, 73.87, H, 6.20, N, 2.97. Found C, 73.77, H, 6.30, N, 2.97.

5ci: methyl 1-oxo-2' -(p-chlorophenyl)-4'-( $p$-methoxyphenyl)-3,4-dihydro-1H-spiro [naphthalene-2, 3'-pyrrolidine]-5'-carboxylate

Yield $0.38 \mathrm{~g}(80 \%)$; yellow crystals, mp: $160^{\circ} \mathrm{C} \pm 2$, IR $(\mathrm{KBr}): 1680,1743,3446 \mathrm{~cm}^{-1},{ }^{1} \mathrm{H}$ NMR: 1.71 (td, $J=5 \mathrm{~Hz}, 13.5 \mathrm{~Hz}, 1 \mathrm{H}, \mathrm{H}-3), 1.89$ (dt, $J=3 \mathrm{~Hz}, 11.5 \mathrm{~Hz}, 1 \mathrm{H}, \mathrm{H}-4), 2.68$ (s, br, 1H, NH), 2.69 (dt, $J=3 \mathrm{~Hz}, 11.5 \mathrm{~Hz}, 1 \mathrm{H}, \mathrm{H}-4), 3.01$ (td, $J=5 \mathrm{~Hz}, 13.5 \mathrm{~Hz}, 1 \mathrm{H}, \mathrm{H}-3$ ), 3.68 
(2s, 6H, OCH 3 ), 4.25 (d, $J=9.3 \mathrm{~Hz}, 1 \mathrm{H}, \mathrm{H}-5$ '), 4.38 (d, J = $9.3 \mathrm{~Hz}, 1 \mathrm{H}, \mathrm{H}-4$ '), 4.53 (s, 1H, H-

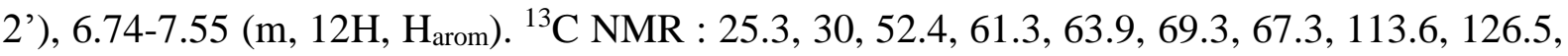
127.6, 128.1, 128.2, 129.2, 129.3, 130.3, 132.8, 133.2, 133.4, 138.2, 141.9, 158.6, 173.9, 198 .Anal. Calcd. for $\mathrm{C}_{28} \mathrm{H}_{26} \mathrm{ClNO}_{4}$ : C, 70.66, H, 5.51, N, 2.94. Found: C, 70.66, H, 5.51, N, 2.94.

5df: methyl 1-oxo-4'-(p-chlorophenyl)-2'-phenyl-3,4-dihydro-1H-spiro[naphthalene2,3'-pyrrolidine]-5'-carboxylate

Yield $0.32 \mathrm{~g}$ (74\%); white crystals, mp: $182^{\circ} \mathrm{C} \pm 2$, IR (KBr): $1675,1744,3447 \mathrm{~cm}^{-1},{ }^{1} \mathrm{H}$ NMR : 1.71 (td, $J=5 \mathrm{~Hz}, 13.8 \mathrm{~Hz}, 1 \mathrm{H}, \mathrm{H}-3), 2.01$ (dt, $J=3 \mathrm{~Hz}, 11.5 \mathrm{~Hz}, 1 \mathrm{H}, \mathrm{H}-4), 2.58$ (s, br, 1H, NH), 2.72 (dt, $J=3 \mathrm{~Hz}, 11.5 \mathrm{~Hz}, 1 \mathrm{H}, \mathrm{H}-4), 3.23$ (td, $J=5 \mathrm{~Hz}, 13.8 \mathrm{~Hz}, 1 \mathrm{H}, \mathrm{H}-3), 3.72$ (s, 3H, $\mathrm{CH}_{3}$ ), 3.91 (d, $J=9 \mathrm{~Hz}, 1 \mathrm{H}, \mathrm{H}-5$ '), 4.25 (d, $J=9 \mathrm{~Hz}, 1 \mathrm{H}, \mathrm{H}-4$ '), 4.40 (s, 1H, H-2'), 7.02-8.20 (m, 13H, $\left.\mathrm{H}_{\text {arom }}\right) .{ }^{13} \mathrm{C}$ NMR: 25.3, 35.5, 52.5, 55.3, 65.5, 65.9, 67.3, 123.4, 123.5, 126.5, 131, 133, 137.8, 141.9, 151.7, 173, 198. Anal. Calcd. for $\mathrm{C}_{27} \mathrm{H}_{24} \mathrm{ClNO}_{3}$ : C, 27.72, $\mathrm{H}$, 5.42, N, 3.14. Found: C, 27.62, H, 5.41, N, 3.12.

\section{5dg: methyl 1-oxo-4'-(p-chlorophenyl)-2'-(p-methylphenyl)-3, 4-dihydro-1H-} spiro[naphthalene-2, 3'-pyrrolidine]-5'-carboxylate

Yield $0.22 \mathrm{~g}(50 \%)$, white crystals, mp: $170^{\circ} \mathrm{C} \pm 2$, IR (KBr):1676, 1745, $3447 \mathrm{~cm}^{-1},{ }^{1} \mathrm{H}$ NMR : 1.74 (td, $J=5 \mathrm{~Hz}, 13.5 \mathrm{~Hz}, 1 \mathrm{H}, \mathrm{H}-3), 1.99$ (dt, $J=3 \mathrm{~Hz}, 12 \mathrm{~Hz}, 1 \mathrm{H}, \mathrm{H}-4), 2.17$ (s, 3H, Ar-CH3), 2.75 (dt, $J=3 \mathrm{~Hz}, 12 \mathrm{~Hz}, 1 \mathrm{H}, \mathrm{H}-4), 2.84$ (s, br, 1H, NH), 3.15 (td, $J=5 \mathrm{~Hz}, 13.5$ $\mathrm{Hz}, 1 \mathrm{H}, \mathrm{H}-3), 3.73\left(\mathrm{~s}, 3 \mathrm{H}, \mathrm{CH}_{3}\right), 4.31$ (d, $J=9.3 \mathrm{~Hz}, 1 \mathrm{H}, \mathrm{H}-5$ '), 4.52 (d, $J=9.3 \mathrm{~Hz}, 1 \mathrm{H}, \mathrm{H}-4^{\prime}$ ), 4.61 (s, 1H, H-2'), 6.84-7.60 (m, 12H, Harom). ${ }^{13} \mathrm{C} \mathrm{NMR}: 21,25.3,30.3,52.4,54.5,61.5$, 63.7, 70.1, 126.3, 127.6, 127.7, 128.7, 130.7, 132.9, 132.9, 133, 136.3, 136.4, 137.5, 141.9, 173.6, 197.9 . Anal. Calcd. for $\mathrm{C}_{28} \mathrm{H}_{26} \mathrm{ClNO}_{3}$ : C, 73.11, H, 5.7, N, 3.05. Found: C, 73.09, H, $5.5, \mathrm{~N}, 3.03$.

5dh: methyl 1-oxo-4' -(p-chlorophenyl)-2' -(p-methoxyphenyl)-3,4-dihydro-1Hspiro[naphthalene-2, 3'-pyrrolidine]-5'-carboxylate

Yield $0.28 \mathrm{~g}(60 \%)$, white crystals, mp: $172{ }^{\circ} \mathrm{C} \pm 2$, IR (KBr):1677, 1745, $3447 \mathrm{~cm}^{-1},{ }^{1} \mathrm{H}$ NMR : 1.64 (td, $J=5 \mathrm{~Hz}, 13.8 \mathrm{~Hz}, 1 \mathrm{H}, \mathrm{H}-3), 1.72$ (dt, $J=3 \mathrm{~Hz}, 11.5 \mathrm{~Hz}, 1 \mathrm{H}, \mathrm{H}-4), 2.82$ (dt, $J$ $=3 \mathrm{~Hz}, 11.5 \mathrm{~Hz}, 1 \mathrm{H}, \mathrm{H}-4), 2.87$ (s, br, $1 \mathrm{H}, \mathrm{NH}), 3.28$ (td, J=5 Hz, $13.8 \mathrm{~Hz}, 1 \mathrm{H}, \mathrm{H}-3), 3.69$ $\left(2 \mathrm{~s}, 6 \mathrm{H}, \mathrm{OCH}_{3}\right), 4.28$ (d, $J=9.3 \mathrm{~Hz}, 1 \mathrm{H}, \mathrm{H}-5$ '), 4.52 (d, $J=9.3 \mathrm{~Hz}, 1 \mathrm{H}, \mathrm{H}-4$ '), 4.59 (s, 1H, H2'), 6.57-7.61 (m, 12H, Harom). ${ }^{13} \mathrm{C}$ NMR: 25.3, 30.4, 52.4, 54.4, 55.2, 61.2, 69.8, 113.4, 126.4, 127.6, 128.1, 128.4, 129, 130.6, 131.3, 132.9, 132.9, 133, 136.4, 141.9, 159.1, 159.1, 173.6, 198.1. Anal. Calcd. for $\mathrm{C}_{28} \mathrm{H}_{26} \mathrm{ClNO}_{4}$ : C, 70.66, H, 5.51, N, 2.94. Found: C, 70.66, H, $5.51, \mathrm{~N}, 2.94$.

\section{5di: methyl 1-oxo-2',4'-di(p-chlorophenyl)-3,4-dihydro-1H-spiro[naphthalene-2,3'-} pyrrolidine]-5'-carboxylate

Yield $0.38 \mathrm{~g}(68 \%)$, white crystals, mp: $188^{\circ} \mathrm{C} \pm 2$. IR $(\mathrm{KBr}): 1675,1744,3447 \mathrm{~cm}^{-1},{ }^{1} \mathrm{H}$ NMR : 1.87 (td, $J=4.5 \mathrm{~Hz}, 13.5 \mathrm{~Hz}, 1 \mathrm{H}, \mathrm{H}-3), 1.93$ (dt, $J=3 \mathrm{~Hz}, 12 \mathrm{~Hz}, 1 \mathrm{H}, \mathrm{H}-4), 2.76$ (dt, $J$ $=3 \mathrm{~Hz}, 12 \mathrm{~Hz}, 1 \mathrm{H}, \mathrm{H}-4), 2.79$ (s, br, $1 \mathrm{H}, \mathrm{NH}), 3.03$ (td, $J=4.5 \mathrm{~Hz}, 13.5 \mathrm{~Hz}, 1 \mathrm{H}, \mathrm{H}-3), 3.59$ $\left(\mathrm{s}, 3 \mathrm{H}, \mathrm{OCH}_{3}\right), 4.25$ (d, $\left.J=9.6 \mathrm{~Hz}, 1 \mathrm{H}, \mathrm{H}-5^{\prime}\right), 4.46$ (d, $\left.J=9.6 \mathrm{~Hz}, 1 \mathrm{H}, \mathrm{H}-4^{\prime}\right), 4.56$ (s, 1H, H2'), 6.94-7.52 (m, 12H, Harom). ${ }^{13} \mathrm{C}$ NMR: 25.2, 29.7, 52.5, 53.7, 61.4, 63.3, 68.9, 126.6, 127.6, 128.1, 128.2, 128.4, 129.2, 130.6, 132.6, 133.0, 133.4, 133.5, 135.7, 138.2, 141.7, 173.5, 197.4. Anal. Calcd. for $\mathrm{C}_{27} \mathrm{H}_{23} \mathrm{Cl}_{2} \mathrm{NO}_{3}$ : C, 67.51, H, 4.83, N, 2.92. Found: C, 67.49, $\mathrm{H}$, $4.85, \mathrm{~N}, 2.92$. 
5ef: methyl 1-oxo-2'-phenyl-4' -(p-nitrophenyl)-3,4-dihydro-1H-spiro[naphthalene2,3'-pyrrolidine]-5'-carboxylate

Yield $0.37 \mathrm{~g}(83 \%)$, beige crystals, mp: $168^{\circ} \mathrm{C} \pm 2$, IR $(\mathrm{KBr}): 1673,1731,3446 \mathrm{~cm}^{-1},{ }^{1} \mathrm{H}$ NMR: 1.79 (td, $J=4.5 \mathrm{~Hz}, 13.5 \mathrm{~Hz}, 1 \mathrm{H}, \mathrm{H}-3), 1.99$ (dt, $J=3 \mathrm{~Hz}, 12.5 \mathrm{~Hz}, 1 \mathrm{H}, \mathrm{H}-4), 2.55$ (dt, $J=3 \mathrm{~Hz}, 12.5 \mathrm{~Hz}, 1 \mathrm{H}, \mathrm{H}-4), 2.67$ (s, br, 1H, NH), 3.11 (td, $J=4.5 \mathrm{~Hz}, 13.5 \mathrm{~Hz}, 1 \mathrm{H}, \mathrm{H}-3)$, 3.62 (s, 3H, OCH 3$), 4.39$ (d, J = 9 Hz, 1H, H-5'), 4.45 (d, $J=9 \mathrm{~Hz}, 1 \mathrm{H}, \mathrm{H}-4$ ') , 4.56 (s, $1 \mathrm{H}, \mathrm{H}-$ 2'), 6.79-7.67 (m, 13H, Harom). ${ }^{13} \mathrm{C}$ NMR: 25.3, 30.5, 52.4, 55.5, 61.3, 64.2, 70.9, 126.3, 127.4, 127.5, 128.1 130.3, 131.5, 137.6, 141.4, 173.8, 198.5. Anal. Calcd. for $\mathrm{C}_{27} \mathrm{H}_{24} \mathrm{~N}_{2} \mathrm{O}_{5}$ : $\mathrm{C}$, 71.04, H, 5.30, N, 6.14. Found: C, 71.06, H, 5.29, N, 6.14.

5eg: methyl 1-oxo-2'-(p-methylphenyl)-4' -(p-nitrophenyl)-3,4-dihydro-1Hspiro[naphthalene-2,3'-pyrrolidine]-5'-carboxylate

Yield $0.23 \mathrm{~g}(51 \%)$, yellow crystals, mp: $168^{\circ} \mathrm{C} \pm 2$, IR $(\mathrm{KBr}): 1677,1735,3446 \mathrm{~cm}^{-1},{ }^{1} \mathrm{H}$ NMR: 1.58 (td, $J=5 \mathrm{~Hz}, 13.5 \mathrm{~Hz}, 1 \mathrm{H}, \mathrm{H}-3), 1.75$ (dt, $J=3 \mathrm{~Hz}, 11.5 \mathrm{~Hz}, 1 \mathrm{H}, \mathrm{H}-4), 2.23$ (s, $\left.3 \mathrm{H}, \mathrm{Ar}-\mathrm{CH}_{3}\right), 2.65$ (dt, $\left.J=3 \mathrm{~Hz}, 11.5 \mathrm{~Hz}, 1 \mathrm{H}, \mathrm{H}-4\right), 2.84$ (s, br, 1H, NH), 2.96 (td, $J=5 \mathrm{~Hz}$, $13.5 \mathrm{~Hz}, 1 \mathrm{H}, \mathrm{H}-3), 3.72\left(\mathrm{~s}, 3 \mathrm{H}, \mathrm{OCH}_{3}\right), 4.33$ (d, J = 9 Hz, 1H, H-5'), 4.39 (d, J = 9 Hz, 1H, H4'), 4.45 (s, 1H, H-2'), 6.72-7.82 (m, 12H, $\mathrm{H}_{\text {arom }}$ ). ${ }^{13} \mathrm{C}$ NMR:25.2, $30.4,52.4,54.2,56.3$, 61.2 , 64.23, 126.5, 127.5, 127.6, 128.1, 128.7, 130.3, 132.6, 123, 133.2, 137.7, $141.6,145.2$ ,173.6 , 198.7. Anal. Calcd. for $\mathrm{C}_{28} \mathrm{H}_{26} \mathrm{~N}_{2} \mathrm{O}_{5}$ : C, 71.47, H, 5.57, N, 5.95. Found: C, 71.47, H, $5.57, \mathrm{~N}, 5.95$.

5eh: methyl 1-oxo-2' -(p-methoxyphenyl)-4' -( $p$-nitrophenyl)-3,4-dihydro-1Hspiro[naphthalene-2,3'-pyrrolidine]-5'-carboxylate

Yield $0.39 \mathrm{~g}(81 \%)$, yellow crystals, $\mathrm{mp}: 170^{\circ} \mathrm{C} \pm 2$, IR (KBr): $1675,1729,3445 \mathrm{~cm}^{-1},{ }^{1} \mathrm{H}$ NMR: 1.57 (td, $J=4.5 \mathrm{~Hz}, 13 \mathrm{~Hz}, 1 \mathrm{H}, \mathrm{H}-3), 1.81$ (dt, $J=3 \mathrm{~Hz}, 11 \mathrm{~Hz}, 1 \mathrm{H}, \mathrm{H}-4), 2.53$ (dt, $J=3 \mathrm{~Hz}, 11 \mathrm{~Hz}, 1 \mathrm{H}, \mathrm{H}-4), 2.79$ (s, br, 1H, NH), 2.98 (td, $J=4.5 \mathrm{~Hz}, 13 \mathrm{~Hz}, 1 \mathrm{H}, \mathrm{H}-3$ ), 3.59 (s, $\left.3 \mathrm{H}, \mathrm{OCH}_{3}\right), 3.64$ (s, 3H, $\left.\mathrm{OCH}_{3}\right), 4.31$ (d, $J=9.8 \mathrm{~Hz}, 1 \mathrm{H}, \mathrm{H}-5$ '), 4.42 (d, J = 9.8 Hz, 1H, H-4') , 4.50 (s,1H, H-2'), 6.78-7.02 (m, 12H, $\left.\mathrm{H}_{\text {arom }}\right) .{ }^{13} \mathrm{C}$ NMR: 24.9, 29.2, 35.4, 51.9, 55.7, 59.3, 64.1, 67.6, 120.9, 121.1, 124.1, 124.5, 125.9, 128.1, 128.6, 129.1, 129.1, 129.2, 129.2, 132.8, 133.0, 134.3, 139.9, 145.4, 145.6, 157.9, 171.6, 198.4. Anal. Calcd. for $\mathrm{C}_{28} \mathrm{H}_{26} \mathrm{~N}_{2} \mathrm{O}_{6}: \mathrm{C}, 69.12$, H, 5.51, N, 2.94. Found: C, 69.11, H, 5.51, N, 2.84 .

5ei: methyl 1-oxo-2'-(p-chlorophenyl)-4'-(p-nitrophenyl)-3,4-dihydro-1Hspiro[naphthalene-2,3'-pyrrolidine]-5'-carboxylate

Yield $0.37 \mathrm{~g}(77 \%)$, beige crystals, $\mathrm{mp}: 171^{\circ} \mathrm{C} \pm 2, \mathrm{IR}(\mathrm{KBr}): 1680,1743,3445 \mathrm{~cm}^{-1},{ }^{1} \mathrm{H}$ NMR :1.57 (td, $J=5 \mathrm{~Hz}, 13.5 \mathrm{~Hz}, 1 \mathrm{H}, \mathrm{H}-3), 2.02$ (dt, $J=3 \mathrm{~Hz}, 11.5 \mathrm{~Hz}, 1 \mathrm{H}, \mathrm{H}-4), 3.21$ (dt, $J$ $=3 \mathrm{~Hz}, 11.5 \mathrm{~Hz}, 1 \mathrm{H}, \mathrm{H}-4), 3.23(\mathrm{~s}, \mathrm{br}, 1 \mathrm{H}, \mathrm{NH}), 3.56(\mathrm{td}, J=5 \mathrm{~Hz}, 13.5 \mathrm{~Hz}, 1 \mathrm{H}, \mathrm{H}-3), 3.52$ (s, 3H, $\left.\mathrm{OCH}_{3}\right), 4.40$ (d, $J=9.5 \mathrm{~Hz}, 1 \mathrm{H}, \mathrm{H}-5^{\prime}$ ), 4.62 (d, $\left.J=9 . \mathrm{Hz}, 1 \mathrm{H}, \mathrm{H}-4^{\prime}\right), 4.68$ (s, 1H, H-2'), 6.93-7.97 (m, 12H, $\mathrm{H}_{\text {arom }}$ ). ${ }^{13} \mathrm{C}$ NMR: 25.2, 29.8, 52.5, 52.7, 61.3, 62.3, 67.8, 124.6, 125.5, $126.3,126.9,127.3,127.8,131.0,131.8,132.1,136.5,142.5,173.2,197.2$. Anal. Calcd. for $\mathrm{C}_{27} \mathrm{H}_{23} \mathrm{ClN}_{2} \mathrm{O}_{5}$ : C, 66.06, H, 4.72, N, 5.71. Found: C, 69.10, H, 5.52, N, 2.82.

6ag: methyl 1-oxo-4'-phenyl-2'-( $p$-methylphenyl)-1,3,4',5'-tetrahydrospiro[indene-2, 3'-pyrrole]-5'-carboxylate

Yield $0.19 \mathrm{~g}(60 \%)$, white needles, mp: $176^{\circ} \mathrm{C} \pm 2$, IR $(\mathrm{KBr}): 1639,1680,1740 \mathrm{~cm}^{-1},{ }^{1} \mathrm{H}$ NMR: 2.09 (s, 3H, Ar-CH $), 2.94-3.12(\mathrm{AB}, 2 \mathrm{H}, J=18 \mathrm{~Hz}, \mathrm{H}-3), 3.73$ (s, 3H, $\left.\mathrm{CH}_{3}\right), 4.10$ (d, $1 \mathrm{H}, J=6 \mathrm{~Hz}, \mathrm{H}-5$ '), 4.91(d, $\left.1 \mathrm{H}, J=6 \mathrm{~Hz}, \mathrm{H}-4^{\prime}\right), 6.77-7.33$ (m, 13H, $\mathrm{H}_{\text {arom }}$ ), ${ }^{13} \mathrm{C}$ NMR: 21.3 , 35.2, 51.4, 55.8, 65.5, 67.4, 72.8, 113.3, 119.4, 125.6, 127.5, 128.1, 128.2, 129.5, 130.1, $131.8,133.3,134.9,134.9,135.8,139,151,170.2$, 175.2, 208.2. Anal. Calcd. for: $\mathrm{C}_{27} \mathrm{H}_{23} \mathrm{NO}_{3}$ : C 79.20, H, 5.66, N; 3.42, Found: C, 79.22, H, 5.66, N, 3.40. 
6ah: methyl 1-oxo-4'-phenyl-2'-(p-methoxyphenyl)-1,3,4',5'-tetrahydrospiro[indene2,3'-pyrrole]-5'-carboxylate

Yield $0.24 \mathrm{~g}(75 \%)$; white crystals, $\mathrm{mp}: 178^{\circ} \mathrm{C} \pm 2$, IR $(\mathrm{KBr}): 1642,1677,1735 \mathrm{~cm}^{-1},{ }^{1} \mathrm{H}$ NMR: 3.72 (s, 3H, Ar-CH CH $_{3}$, 2.95-3.28 (AB, 2H, J = $\left.18 \mathrm{~Hz}, \mathrm{H}-3\right), 3.73$ (s, 3H, $\left.\mathrm{OCH}_{3}\right), 4.2$ (d, $1 \mathrm{H}, J=6 \mathrm{~Hz}, \mathrm{H}-5$ '), 5.16 (d, $1 \mathrm{H}, J=6 \mathrm{~Hz}, \mathrm{H}-4$ ') $, 6.51-7.34\left(\mathrm{~m}, 13 \mathrm{H}, \mathrm{H}_{\text {arom }}\right),{ }^{13} \mathrm{C} \mathrm{NMR}: 21.3$, $35.2,52.4,57.8,69.9,67.4,75.3,124.6,127.5,128.1,128.2,129.5,130.1,131.8,133.3$, 134.9, 134.9, 135.8, 139, 152.1, 171.5, 175.3, 205.2. Anal. Calcd. for $\mathrm{C}_{27} \mathrm{H}_{23} \mathrm{NO}_{4}: \mathrm{C}, 76.22$, H, 5.45, N, 3.29, Found: C, 76.24, H, 5.47, N, 3.27.

6df: methyl 1-oxo-4'-(p-chlorophenyl)-2'-phenyl-1,3,4',5'-tetrahydrospiro[indene2,3'-pyrrole]-5'-carboxylate

Yield $0.19 \mathrm{~g}(65 \%)$; white needles, $\mathrm{mp}: 160^{\circ} \mathrm{C} \pm 2$, IR $(\mathrm{KBr}): 1642,1677,1735 \mathrm{~cm}^{-1},{ }^{1} \mathrm{H}$ NMR: 2.90-3.02 (AB, 2H, $J=18 \mathrm{~Hz}, \mathrm{H}-3), 3.8$ (s, 3H, $\left.\mathrm{OCH}_{3}\right), 4.3$ (d, 1H, $J=6 \mathrm{~Hz}, \mathrm{H}-5$ '), $5.15\left(\mathrm{~d}, 1 \mathrm{H}, J=6 \mathrm{~Hz}, \mathrm{H}-4{ }^{\prime}\right), 6.96-7.50\left(\mathrm{~m}, 13 \mathrm{H}, \mathrm{H}_{\text {arom }}\right),{ }^{13} \mathrm{C} \mathrm{NMR}: 32.6,52.5,55.1,55.3,65.6$, 70.4, 75.6, 123.3, 125.3, 127.5, 128.3, 129.2, 129.5, 130.5, 132.9, 133.5, 134.9, 134.9, 135.8, 139.2, 151.6, 171.4, 175.5, 207.5, Anal. Calcd. for $\mathrm{C}_{26} \mathrm{H}_{20} \mathrm{ClNO}_{3}$ : C, 72.64, H, 4.69, N, 3.26, Found: C, 72.62, H, 4.66, N, 3.23.

\section{X-ray crystallography}

Colourless crystals of $\mathbf{4 b g}, \mathbf{4 c i}$ and $\mathbf{5 b i}$ have been mounted on a Nonius Kappa Apex II diffractometer and the intensity data have been collected at $115 \mathrm{~K}$ with Mo $\mathrm{K}_{\alpha}$ radiation of $\lambda=0.71073 \AA$. These data were further treated with the suite of SAINT V8.27B (Bruker AXS Inc., 2012) programs and within the OLEX 2 frame $^{34}$. The models of the structures have been solved by direct methods with SUPERFLIP ${ }^{35}$ (4bg and 4ci) or with SHELXS-97 ${ }^{30}$ (5bi) and refined with SHELXL- $97^{36}$. There is a slight disorder of the OMe group in 5bi with statistic occupancies of 0.56 and 0.44 for $\mathrm{O}, \mathrm{C}$ and $\mathrm{H}$ atoms of this group. All non-hydrogen atoms were refined with anisotropic temperature factors. The hydrogens bound to $\mathrm{N}$ atoms were located from difference-Fourier maps and isotropically refined for $\mathbf{4 b g}$ and $\mathbf{4 c i}$ but included in a riding model for $\mathbf{5 b i}$. All other $\mathrm{H}$ atoms were placed in calculated positions and refined as riding on the heavy atoms bearing them.

Crystallographic data (excluding structure factors) have been deposited with the Cambridge Crystallographic Data Centre: deposition numbers CCDC 1029334 (4bg), CCDC 1029335 (4ci) and CCDC 1029336 (5bi) contain detailed crystallographic data for this publication. These data may be obtained free of charge from the Cambridge Crystallographic Data Center through www.ccdc.cam.ac.uk/data_request/cif.

\section{References}

1- (a) K. V. Gothelf and K. A. Jorgensen, Chem. Rev., 1998, 98, 863-909. (b) D. Gavaskar, R. Raghunathan and A. R. Suresh Babu, Tetrahedron Lett., 2014, 55, 2217-2220. (c) A. Padwa (Ed.), In 1, 3-Dipolar Cycloaddition Chemistry; Wiley, New York, 1984. (d) P. S. Yuvaraj and B. S. R. Reddy, Tetrahedron Lett., 2013, 54, 821-827.

2- (a) C. P. Dell, J. Chem. Soc., Perkin Trans 1, 1998, 3873-3905. (b) W. Carruthers, In Cycloaddition Reactions in Organic Synthesis; Pergamon Press, Oxford, 1990.

3- (a) M. Bakthadoss, N. Sivakumar, A. Devaraj and D. S. Sharada, Synthesis, 2011, 13, 2136-2146. (b) A. R. Suresh Babu, D. Gavaskar and R. Raghunathan, J. Organomet. Chem., 2013, 745-746, 409-416. 
4- (a) F. J. Sardina and H. Rapopport, Chem. Rev., 1996, 96, 1825-1872. (b) J. Seyden-Penne, In Chiral Auxiliaries and Ligands in Asymmetric Synthesis; Wiley, New York, 1996.

5- S. Kanemasa and O. Tsuge, In Advances in Cycloaddition, ed. by D. P. Curran; Jai Press: Greenwich, 1993, Vol. 3, pp. 99-159.

6- (a) S. K. Dey, D. Bose, A. Hazra, S. Naskar, A. Nandy, R. N. Munda, S. Das, N. Chatterjee, N. B. Mondal, S. Banerjee and K. D. Saha, PLos One, 2013, 8, 58055.

(b) A. Kumar, G. Gupta, S. Srivastava, A. K. Bishnoi, R. Saxena, R. Kant, R. S. Khanna, P. R. Maulik and A. Dwivedi, R. Soc. Chem. Adv., 2013, 3, 4731-4735.

7- (a) G. Periyasami, R. Raghunathan, G. Surendiran and N. Mathivanan, Eur. J. Med. Chem., 2009, 44, 959-966. (b) A. Mendoza, S. Pérez-Silanes, M. Quiliano, A. Pabón, S. Galiano and G. González, Exp. Parasitol., 2011, 128, 97-103.

8- (a) N. Arumugam, G. Periyasami, R. Raghunathan, S. Kamalraj and J. Muthumary, Eur. J. Med. Chem., 2011, 46, 600-607. (b) G. Chen, H-P. He, J. Ding and X-J. Hao, Heterocycl. Commun., 2011, 15, 355-360.

9- (a) R. Grigg, Chem. Soc. Rev., 1987, 16, 89-121. (b) J. W. Lowin, In 1, 3- Dipolar Cycloaddition Chemistry; ed. by A. Padwa; J. Wiley \& Sons: NewYork, 1984; Vol. 1, pp. 653-670. (c) O. Tsuge and S. Kanemasa, In Advances in Heterocyclic Chemistry; ed. by A. R. Katritzky; Academic Press: San Diego, 1989, Vol. 45, pp. 232-349. (d) E. Vedejs, In Advances in Cycloaddition; ed. by D. P. Curran; Jai Press: Greenwich, 1988, Vol. 1, pp. 351-384.

10- R. Grigg and V. Sridharan, In Advances in Cycloaddition; ed. by D. P. Curran; Jai Press: Greenwich, 1993, Vol. 3, pp. 161-204.

11- (a) G. Subramaniyan and R. Raghunathan, Tetrahedron, 2001, 57, 2909-2913. (b) G. Subramaniyan, R. Raghunathan and A. M. M. Castro, Tetrahedron, 2003, 59, 335-340, (c) M. Nyerges, I. Fejes and L. Töke, Tetrahedron Lett. 2000, 41, 7951-7954.

12- A. I. Almansour, R. S. Kumar, F. Beevi, A. N. Shirazi, H. Osman, R. Ismail, T. S. Choon, B. Sullivan, K. McCaffrey, A. Nahhas, K. Parang and M. A. Ali, Molecules, 2014, 19, 10033-10055.

13- K. Krzysztof, O. Jolanta and D. Malgorzata, Eur. J. Med. Chem., 2008, 43, 53-61.

14- (a) W.-L. Wang, T.-J. Zhu, H.-W. Tao, Z.-Y. Lu, Y.-C. Fang, Q.-Q. Gu and W.-M. Zhu. Chem. Biodivers., 2007, 4, 2913-2919. (b) P. Hyeong-Beom, J.N. Hyun, H.J. Hee, C.J. Hoon, C. Jung-Hyuck and Y.K. Ho. Arch. Pharm. Chem., 2007, 340, 530-537.

15- (a) H. Schumann, O. Stenzel and F. Girgsdies, Organometallics, 2001, 20, 1743-1751. b) M. N. Herzog, J. C. W. Chien and M. D. Rausch, J. Organomet. Chem., 2002, 654, 29-35.

16- R. Sheng, Y. Xu, C. Hu, J. Zhang, X. Lin, J. Li, B. Yang, Q. He and Y. Hu, Eur. J. Med. Chem., 2009, 44, 7-17.

17- H. O. Saxena, U. Faridi, Bioorg. Med. Chem. Lett., 2008, 18, 3914-3918.

18- (a) M. S. Novikov, A. F. Khlebnikov, E. S. Sidorina, A. E. Masalev, J. Kopf and R. R. Kostikov, Russ. J. Org. Chem., 2002, 38, 672-682. (b) C. Nájera and J. M. Sansano. Top. Heterocycl. Chem., 2008, 12, 117-145. (c) G. Cao, F. Long, Y. Zhao, Y. Wang, L. Huang, D. Teng, Tetrahedron, 2014, 70, 9359-9365. (d) K. Shimizu, K. Ogata and S.-I. Fukuzawa, Tetrahedron Lett., 2010, 51, 5068-5070.(e) N. Fernández, L. Carrillo, J. L. Vicario, D. Badía and E. Reyes, Chem. Commun., 2011, 47, 12313-12315 (f) A. P. Kadina, A. F. Khlebnikov, M. S. Novikov, P. J. Pérez and D. S. Yufit, Org. Biomol. Chem., 2012, 10, 5582-5591 (g) G. A. Oliveira Udry, E. Repetto and O. Varela, J. Org. Chem., 2014, 79, 4992-5006. 
19- T. L. Liu, Z. L. He, Q. H. Li, H. Y. Tao and C. J. Wang, Adv. Synth. Catal., 2011, 353, $1713-1719$.

20- N. Wannassi, M. M. Rammah, S. Boudriga, M. B. Rammah, K. M. Jobé, K. Ciamala, M. Knorr, M. Enescu, Y. Rousselin and M. M. Kubicki, Heterocycles, 2010, 81, 2749-2762.

21- M. Askri, N. Jgham, M. Rammah, K. Ciamala, K. Monnier-Jobé, and J. Vebrel, Heterocycles, 2007, 71, 289-303.

22- M. Askri, N. Ben Hamadi, M. Msadek, and M. E. Rammah, J. Soc. Chim. Tunisie, 2006, $8,219-222$.

23- (a) S. Haddad, I. Jalloul, S. Boudriga, M. Askri, M. Knorr, Y. Rousselin and M. M. Kubicki, J. Soc. Chim. Tunisie, 2013, 15, 1-8. (b) S. Haddad, S. Boudriga, F. Porzio, A. Soldera, M. Askri, D. Sriram, P. Yogeeswari, M. Knorr, Y. Rousselin and M. M. Kubicki, RSC Adv., 2014, 4, 59462-59471. (c) S. Haddad, S. Boudriga, T. N. Akhaja, J. P. Raval, F. Porzio, A. Soldera, M. Askri, M. Knorr, Y. Rousselin, M. M. Kubicki and D. Rajani, New J. Chem., 2015, 39, 520-528

24- M. Mssadek, M. Rammah, G. Schmitt and J. Vebrel, Bull. Soc. Chim. Belg., 1992,101, 323-327.

25- D. M. Cooper, R. Grigg, S. Hargreaves; P. Kennewell and J. Redpath, Tetrahedron, 1995, 51, 7791-7808.

26- R. Grigg, D. M. Cooper, S. Holloway, S. McDonald, E. Millington and M.A. B. Sarker, Tetrahedron, 2005, 61, 8677-8685.

27- S. Cabrera, R. G. Arrayas, B. M. Matute; F. P. Coss and J. Caretero, Tetrahedron, 2007, 63, 6587-6602.

28- (a) O. Dogan, H. Koyuncu and U. Kaniskan, Turk. J. Chem., 2001, 25, 365-371. (b) J. M. Longmire, B. Wang and X. Zhang. J. Am. Chem. Soc., 2002, 124, 13400-13401. (c) K. V. Kudryavtsev and A. A. Zagulyaeva, Russ. J. Org. Chem., 2008, 44, 378-387.

29- (a) J. Casas, R. Grigg, C. Najera and J.M. Sansano, Eur. J. Org. Chem., 2001, 1971-1982.

(b) C. Nájera and J.M. Sansano, J. Organomet. Chem., 2014, 771, 78-92.

30- J. C. Pelletier and M. P. Cava, J. Org. Chem., 1987, 52, 616-622.

31- X. Yu, Y. Yang, X. Zou, Hongwen Tao, Y. Ling, Q. Yao, H. Zhou and P. Yi, Spectrochim. Acta Part A, 2012, 94, 23-29.

32- D .Magde, J. H. Brannon, T. L. Cremers and J. Olmsted, J. Phys. Chem., 1979, 83, 696-699. H. Maas, A. Khatyr and G. Calzaferri, Micropor. Mesopor. Mater., 2003, 65, 233-242.

33- (a) K. Nasu, T. Nakagawa, H. Nomura, C-J. Lin, C-H. Cheng, M-R. Tseng, T. Yasuda and C. Adachi, Chem. Comm., 2013, 49, 10385-10387. (b) A. Kundu, S. Pathak and A. Pramanik, Asian J. Org. Chem., 2013, 2, 869-876.

34- O. V. Dolomanov, L. J. Bourhis, R. J. Gildea, J. A. K. Howard and H. Puschmann, J. Appl. Cryst., 2009, 42, 339-341.

35- L. Palatinus and G. Chapuis, J. Appl. Cryst., 2007, 40, 786-790.

36- G. M. Sheldrick, Acta Cryst., 2008, A64, 112-122. 